\title{
NUMERICAL APPROXIMATION OF FRACTIONAL POWERS OF ELLIPTIC OPERATORS
}

\author{
ANDREA BONITO AND JOSEPH E. PASCIAK
}

\begin{abstract}
We present and study a novel numerical algorithm to approximate the action of $T^{\beta}:=L^{-\beta}$ where $L$ is a symmetric and positive definite unbounded operator on a Hilbert space $H_{0}$. The numerical method is based on a representation formula for $T^{-\beta}$ in terms of Bochner integrals involving $\left(I+t^{2} L\right)^{-1}$ for $t \in(0, \infty)$.

To develop an approximation to $T^{\beta}$, we introduce a finite element approximation $L_{h}$ to $L$ and base our approximation to $T^{\beta}$ on $T_{h}^{\beta}:=L_{h}^{-\beta}$. The direct evaluation of $T_{h}^{\beta}$ is extremely expensive as it involves expansion in the basis of eigenfunctions for $L_{h}$. The above mentioned representation formula holds for $T_{h}^{-\beta}$ and we propose three quadrature approximations denoted generically by $Q_{h}^{\beta}$. The two results of this paper bound the errors in the $H_{0}$ inner product of $T^{\beta}-T_{h}^{\beta} \pi_{h}$ and $T_{h}^{\beta}-Q_{h}^{\beta}$ where $\pi_{h}$ is the $H_{0}$ orthogonal projection into the finite element space. We note that the evaluation of $Q_{h}^{\beta}$ involves application of $\left(I+\left(t_{i}\right)^{2} L_{h}\right)^{-1}$ with $t_{i}$ being either a quadrature point or its inverse. Efficient solution algorithms for these problems are available and the problems at different quadrature points can be straightforwardly solved in parallel. Numerical experiments illustrating the theoretical estimates are provided for both the quadrature error $T_{h}^{\beta}-Q_{h}^{\beta}$ and the finite element error $T^{\beta}-T_{h}^{\beta} \pi_{h}$.
\end{abstract}

\section{Introduction.}

The mathematical study of integral or nonlocal operators has received much attention due to their wide range of applications, see for instance $[8,2,11,4,19$, $12,25,29,9]$. A prototype for such nonlocal operator results from the fractional powers of an elliptic operator and is the focus of this paper. A canonical example is given by $L^{\beta}=(I-\Delta)^{\beta}$ on $\mathbb{R}^{d}$ and is naturally defined via Fourier transform, i.e.,

$$
\mathcal{F}\left(L^{\beta} f\right)(\zeta)=\mathcal{F}\left((I-\Delta)^{\beta} f\right)(\zeta)=\left(1+|\zeta|^{2}\right)^{\beta} \mathcal{F}(f)(\zeta) .
$$

Here $\mathcal{F}(v)$ denotes the Fourier transform of $v$ and $\beta$ is any real number.

We shall be concerned with similar problems, but posed on a bounded Lipschitz domain $\Omega$. Specifically, given $f$, we seek $u=L^{-\beta} f$ with $\beta \in(0,1)$ and $L$ given by, for example, $L=(I-\Delta)$. In this case, we need to augment the problem with boundary conditions, e.g.,

$$
\begin{aligned}
\frac{\partial u}{\partial n} & =0, & & \text { on } \Gamma_{N} \subset \partial \Omega, \\
u & =0, & & \text { on } \Gamma_{D}:=\partial \Omega \backslash \Gamma_{N} .
\end{aligned}
$$

Date: May 14, 2018.

2010 Mathematics Subject Classification. 35S15, 65R20, 65N12, 65N50, 65N30. 
Here $n$ denotes the outward pointing normal on $\Gamma_{N}$. When $\Gamma_{D}$ has positive measure, we may also consider $L=-\Delta$.

We are concerned with the numerical approximation of $u:=L^{-\beta} f$. This problem has been studied by numerous authors $[7,14,18,16,28,21,22,31]$ (we give more details below). We note that $L^{-\beta}$ is defined in terms of the spectral decomposition of $L$, i.e, $u:=L^{-\beta} f$ is given by

$$
u(x)=\sum_{i=1}^{\infty} \lambda_{i}^{-\beta} c_{i} \psi_{i}(x) .
$$

Here $c_{i}$ are the Fourier coefficients of $f$ in the $L_{2}$-orthonormal basis $\left\{\psi_{i}(x)\right\}_{i=1}^{\infty}$ of eigenfunctions for $L$ and $\left\{\lambda_{i}\right\}_{i=1}^{\infty} \subset \mathbb{R}_{+} \backslash\{0\}$ are the respective eigenvalues.

There are several techniques for approximating $L^{-\beta} f$ in the literature. The most natural approach is to introduce a (finite dimensional) numerical approximation $L_{h}$ of $L$ and obtain an approximation $u_{h}:=L_{h}^{-\beta} f$ using an expression similar to (2) but involving the eigenfunctions and eigenvalues of $L_{h}[22,21,31]$. The difficulty with this approach is that the direct evaluation of the resulting approximation is computationally very expensive. Indeed, it requires the computation of all eigenvectors and eigenvalues of a large system of linear equations. The purpose of this paper is to provide a more efficient technique for approximating $u_{h}$ (and hence $u$ ) which avoids the above mentioned eigenvalue/eigenvector computations.

An alternative approach for approximating $u:=L^{-\beta} f:=(-\Delta)^{-\beta} f$ (or a more general elliptic partial differential equation) is based on a representation of $u$ via a "Neumann to Dirichlet" map [7]. Namely, $u$ is given, up to a normalization constant, by $u(x)=v(x, 0)$ where $v: \Omega \times \mathbb{R}_{+} \rightarrow \mathbb{R}$ solves

$$
-\operatorname{div}\left(y^{1-2 \beta} \nabla v(x, y)\right)=0, \quad(x, y) \in \Omega \times \mathbb{R}_{+}
$$

together with the boundary conditions:

$$
\begin{gathered}
v(\cdot, y) \text { satisfies }(1) \text { for each } y \in \mathbb{R}_{+}, \\
\lim _{y \rightarrow \infty} v(x, y)=0, \quad x \in \Omega, \\
\lim _{y \rightarrow 0^{+}}\left(-y^{1-2 \beta} v_{y}(x, y)\right)=f(x), \quad x \in \Omega .
\end{gathered}
$$

The above equations leads to a well posed variational problem in an appropriately weighted Sobolev space [28] which is amendable to finite element approximation. The numerical algorithm proposed and analyzed in [28] consists of a $\mathbb{R}^{d+1}$ finite element method which takes advantage of the rapid decay of the solution $v$ in the $y$ direction enabling truncation to a bounded domain of modest size. Error estimates are derived in a weighted $H^{1}(\Omega)$-norm.

Another natural approach for representing $u:=L^{-\beta} f$ is via the Dunford Taylor integral, i.e.,

$$
u=\frac{1}{2 \pi i}\left(\int_{\gamma} z^{-\beta} R_{z}(L) d z\right) f .
$$

Here $\gamma$ is a positively oriented simple closed curve (in the extended complex plane) enclosing the spectrum of $L$ and $R_{z}(L)=(z I-L)^{-1}$ is the resolvent. A quadrature approximation of the above integral is proposed in [16]. In fact, the quadrature proposed in [16] but based on the hyperbolic path given in [17] leads to an exponentially convergent quadrature approximation. Our third scheme below is an example of 
another exponentially convergent quadrature but, in our case, only involves multiple evaluations of $\left(I+t_{i} L\right)^{-1} f$ where $t_{i} \in(0, \infty)$ is related to the quadrature node. There are numerous other papers proposing exponentially convergent quadrature schemes for Dunford-Taylor integral representations of $e^{-t L}$, e.g., $[26,15,16]$.

The algorithm developed here is also based on an integral representation of $u=L^{-\beta} f$ :

$$
\begin{aligned}
L^{-\beta} & =\frac{2 \sin (\pi \beta)}{\pi}\left[\int_{0}^{\infty} t^{2 \beta-1}\left(I+t^{2} L\right)^{-1} d t\right] \\
& =\frac{2 \sin (\pi \beta)}{\pi}\left[\int_{0}^{1} t^{2 \beta-1}\left(I+t^{2} L\right)^{-1} d t+\int_{0}^{1} t^{1-2 \beta}\left(t^{2} I+L\right)^{-1} d t\right],
\end{aligned}
$$

We derive this identity in Section 2 from an identity in [3] (see, also [5, Chapter 10.4] and [32, Chapter 9.11]).

We develop and analyze two different quadrature schemes for the last two integrals above and a third for the first expression on the right of (4) under the transformation $y=\ln (t)$, i.e.,

$$
L^{-\beta}=\frac{2 \sin (\pi \beta)}{\pi}\left[\int_{-\infty}^{\infty} e^{2 \beta y}\left(I+e^{2 y} L\right)^{-1} d y\right] .
$$

The first quadrature is based on a $\beta$-dependent graded mesh of $N$ subintervals per integral and a one point (rectangle) quadrature on each subinterval. Theorem 3.5 shows that there exists a constant $C_{1}$ only depending on $\beta$ and the smallest eigenvalue of $L$ such that

$$
\left\|\left(L_{h}^{-\beta}-\mathcal{Q}_{R, h}^{\beta}\right) f\right\|_{L^{2}(\Omega)} \leq C_{1} N^{-1}\|f\|_{L^{2}(\Omega)},
$$

where $\mathcal{Q}_{R, h}^{\beta}$ is the rectangle quadrature approximation to (4) with $L$ replaced by $L_{h}$, a finite dimensional approximation of $L$. The second quadrature method starts with a dyadic partition of the interval $[0,1]$. Each subinterval is further decomposed in $N$ intervals of the same length on which a $r$ point weighted Gaussian quadrature is applied. Overall, this quadrature algorithm uses a number of quadrature points proportional to $r^{2} N \ln N$ points. Theorem 3.5 shows that there exists a constant $C_{2}$ only depending on $\beta$ and the smallest eigenvalue of $L$ such that

$$
\left\|\left(L_{h}^{-\beta}-\mathcal{Q}_{G, h}^{\beta}\right) f\right\|_{L^{2}(\Omega)} \leq C_{2} N^{-2 r}\|f\|_{L^{2}(\Omega)} .
$$

Here $\mathcal{Q}_{G, h}^{\beta}$ is the Gaussian quadrature approximation of (4) with $L$ replaced by $L_{h}$. The final quadrature involves $2 N+1$ equally spaced points applied to the integral (5) with a mesh size $k=1 / \sqrt{N}$ and yields an approximation $\mathcal{Q}_{E, h}^{\beta}$ satisfying

$$
\left\|\left(L_{h}^{-\beta}-\mathcal{Q}_{E, h}^{\beta}\right) f\right\|_{L^{2}(\Omega)} \leq C_{3} e^{-c_{4} / k}\|f\|_{L^{2}(\Omega)} .
$$

Here $C_{3}$ is a constant only depending on $\beta$ and the smallest eigenvalue of $L$ and $c_{4}$ is an absolute constant. In what follows we denote $\mathcal{Q}_{R, h}^{\beta}, \mathcal{Q}_{G, h}^{\beta}$ or $\mathcal{Q}_{E, h}^{\beta}$ generically by $\mathcal{Q}_{h}^{\beta}$.

The final result provides estimates for $\left\|\left(L^{-\beta}-L_{h}^{-\beta}\right) f\right\|_{L^{2}(\Omega)}$ when $L_{h}^{-1} f$ is a finite element approximation of $L^{-1} f$. These estimates depend on the elliptic regularity index $0<\alpha \leq 1$ (see conditions (c) and (d) of Section 4), e.g., $\alpha=1$ when $\Omega$ is convex, the coefficients of $L$ are smooth and $\Gamma_{D}=\emptyset$ or $\Gamma_{N}=\emptyset$. Theorem 4.3 shows 
that under these conditions, there exists a constant $C_{5}$ uniform in $h$ and such that

$$
\begin{aligned}
& \left\|\left(L^{-\beta}-L_{h}^{-\beta} \pi_{h}\right) f\right\|_{L_{2}(\Omega)} \leq C_{5} h^{2 \alpha} \ln (1 / h) \quad \text { or } \\
& \left\|\left(L^{-\beta}-L_{h}^{-\beta} \pi_{h}\right) f\right\|_{L_{2}(\Omega)} \leq C_{5} h^{2 \alpha}
\end{aligned}
$$

depending on the smoothness of $f$. Here $\pi_{h}$ is now the $L^{2}(\Omega)$ projection onto the finite element subspace. Combining (6), (7) or (8) with (9) provides an estimate for the difference between $L^{-\beta} f$ and its numerical approximation $Q_{h}^{\beta} f$ (see Corollary 4.4). Although the second result of (9) is well known when $\alpha=1$ and $h^{2 \alpha}$ is replaced by $h^{2 \beta}$ (see [13] and Remark 4.3), we believe that our optimal order results are not available.

The computation of $Q_{h}^{\beta} f$ involves multiple independent evaluations of $(I+$ $\left.\left(t_{i}\right)^{2} L_{h}\right)^{-1} f$ with $t_{i} \in(0, \infty)$ which can be implemented trivially in parallel. These are just finite element approximations to the problem $\left(I+\left(t_{i}\right)^{2} L\right) w=f$ (argumented with boundary conditions) and hence their implementation is classical. Moreover, multigrid preconditioners are available whose convergence rates can be guaranteed to be bounded independently of the parameters $t_{i}$ and $h$.

For most of this work, we provide our analysis in a more general framework, namely, we assume that we are given two Hilbert spaces $H^{1} \subset H^{0}$ with $H^{1}$ compactly, continuously and densely embedded in $H^{0}$. Denoting $H^{-1}$ and $\left(H^{0}\right)^{\prime}$ to be the respective duals leads to an operator $T: H^{-1} \rightarrow H^{1}$ (its inverse is $L$ in the above discussion). The usual identification $H^{1} \subset H^{0} \cong\left(H^{0}\right)^{\prime} \subset H^{-1}$ enables the definition of $T^{\beta}$ (see Section 2 for the precise setting). This more general setting contains a large class of operators $T$, for example, the inverse of the Laplace-Beltrami operator defined over Riemannian surfaces.

The outline of the remainder of the paper is as follows. In the next section, we motivate the integral identity (4). Section 3 defines our three numerical integration schemes for the scalar version of (4) (see (17)) and provides bounds for the quadrature error. The main result of this section is Theorem 3.2. This theorem is also illustrated by numerical experiments. The space discretization is developed in Section 4 for general elliptic operators. Theorem 4.3 provides the a-priori error estimate (9). The theoretical results are again followed by numerical illustration.

\section{Integral Representation of Fractional Powers}

We consider two Hilbert spaces $H^{1}$ and $H^{0}$ with inner products and norms, $\langle\cdot, \cdot\rangle_{i}$ and $\|\cdot\|_{i}, i=0,1$, respectively, and make the following two assumptions:

(a) $H^{1}$ is compactly and densely contained in $H^{0}$.

(b) There is a constant $c_{0}$ satisfying

$$
\|u\|_{0} \leq c_{0}\|u\|_{1}, \quad \text { for all } u \in H^{1} .
$$

Let $H^{-1}$ denote the dual space of $H^{1}$ with norm

$$
\|F\|_{-1}=\sup _{\phi \in H^{1}} \frac{\langle F, \phi\rangle}{\|\phi\|_{1}},
$$

where $\langle\cdot, \cdot\rangle$ denotes the duality pairing. We define $T: H^{-1} \rightarrow H^{1}$ by $T F:=u$ where for $F \in H^{-1}, T F \in H^{1}$ is the unique solution of

$$
\langle T F, \phi\rangle_{1}=\langle F, \phi\rangle, \quad \text { for all } \phi \in H^{1} .
$$


It is clear that $T$ is a isomorphism of $H^{-1}$ onto $H^{1}$ and we denote $L$ to be its inverse. We define

$$
D(L):=\left\{v \in H^{0}: L v \in H^{0}\right\} .
$$

Our goal is to use (4) to approximate the fractional powers of $T$, specifically, $T^{\beta} f:=L^{-\beta} f$ for $0<\beta<1$ and $f \in H^{0}$. For convenience we rewrite these integrals as

$$
\begin{aligned}
T^{\beta} & =C_{\beta}^{-1} \int_{0}^{\infty} t^{2 \beta-1} T_{1}(t) d t \\
& =C_{\beta}^{-1}\left[\int_{0}^{1} t^{2 \beta-1} T_{1}(t) d t+\int_{0}^{1} t^{1-2 \beta} T_{2}(t) d t\right]
\end{aligned}
$$

with

$$
C_{\beta}:=\int_{0}^{\infty} t^{2 \beta-1}\left(1+t^{2}\right)^{-1} d t=\frac{\pi}{2 \sin (\pi \beta)}
$$

(The last equality can be verified using the Beta function and the Euler reflexion formula). Here $T_{2}(t):=t^{-2} T_{1}\left(t^{-1}\right)$ and $T_{1}(t): H^{-1} \rightarrow H^{1}$ is defined by $T_{1}(t) F:=u$ with $u \in H^{1}$ solving

$$
\langle u, \phi\rangle_{0}+t^{2}\langle u, \phi\rangle_{1}=\langle F, \phi\rangle, \quad \text { for all } \phi \in H^{1} .
$$

As usual, the function $f \in H^{0}$ is identified with the functional $F \in H^{-1}$ is defined by $\langle F, \phi\rangle=\langle f, \phi\rangle_{0}$, for all $\phi \in H^{1}$. We view (11) as an equality between elements of $B\left(H^{0}, H^{0}\right)$, the set of bounded operators on $H^{0}$.

We note that

$$
\left\|T_{1}(t) f\right\|_{0} \leq\|f\|_{0}
$$

follows immediately from (13). As $u:=T_{2}(t) f$ is the solution of

$$
t^{2}\langle u, \phi\rangle_{0}+\langle u, \phi\rangle_{1}=\langle f, \phi\rangle_{0}, \quad \text { for all } \phi \in H^{1},
$$

we have from (10) that

$$
\|u\|_{0} \leq c_{0}\|u\|_{1} \leq c_{0}\|f\|_{-1} .
$$

It follows, invoking (10) again, that for $f \in H^{0}$,

$$
\|f\|_{-1} \leq c_{0}\|f\|_{0}
$$

and hence

$$
\left\|T_{2}(t) f\right\|_{0}=\|u\|_{0} \leq c_{0}^{2}\|f\|_{0} .
$$

To make sense out of (11), we derive additional properties of $T$. The compactness of $H^{1}$ in $H^{0}$ and (15) immediately imply that $T: H^{-1} \rightarrow H^{-1}$ is compact. Moreover, the norm on $H^{-1}$ comes from the inner product

$$
\langle F, G\rangle_{-1}=\langle F, T G\rangle, \quad \text { for all } F, G \in H^{-1}
$$

from which it immediately follows that $T$ is a symmetric and positive definite operator on $H^{-1}$. Fredholm theory implies that there is an $\langle\cdot, \cdot\rangle_{-1}$ orthonormal basis of eigenfunctions $\left\{\psi_{i}\right\}, i=1,2, \ldots$ for $H^{-1}$. The corresponding (real) eigenvalues $\left\{\mu_{i}\right\}$ are positive and, along with their eigenvectors, can be reordered to be non-increasing with limit 0 . Given $F \in H^{-1}$, we define

$$
T^{\beta} F:=\sum_{i=1}^{\infty} \mu_{i}^{\beta}\left\langle F, \psi_{i}\right\rangle_{-1} \psi_{i} .
$$


The functions $\widetilde{\psi}_{i}=\mu_{i}^{1 / 2} \psi_{i}, i=1,2, \ldots$, provide an orthonormal basis for $H^{0}$ (see Proposition 4.1 below) so that

$$
T^{\beta} f=\sum_{i=1}^{\infty} \mu^{\beta}\left\langle f, \widetilde{\psi}_{i}\right\rangle_{0} \widetilde{\psi}_{i}, \quad \text { for all } f \in H^{0} .
$$

Moreover,

$$
D(L):=\left\{u \in H^{0}: L u \in H^{0}\right\}=\left\{u \in H^{0}: \sum_{i=1}^{\infty} \mu_{i}^{-2}\left\langle u, \widetilde{\psi}_{i}\right\rangle_{0}<\infty\right\} .
$$

This implies that $L$ is a closed operator on $H^{0}$ with domain $D(L)$ and that the range of $L$ on $D(L)$ coincides with $H^{0}$. We then have the following theorem.

Theorem 2.1. Assume that $H^{1}$ and $H^{0}$ are Hilbert spaces satisfying (a) and (b). Then for $0<\beta<1$, the integrals appearing in (11) are Bochner integrable and the equalities hold in $B\left(H^{0}, H^{0}\right)$.

Proof. We note that if $z=(\lambda+L)^{-1} x$ for $\lambda>0$ and $x \in H^{0}$,

$$
\|z\|_{0} \leq \lambda^{-1}\|x\|_{0} \text {. }
$$

This implies (cf. [3], [5, Chapter 10.4] and [32, Chapter 9.11]) that for $\alpha \in(0,1)$ and $x \in D(L)$, the following is a well defined Bochner integral and satisfies

$$
\frac{\sin (\alpha \pi)}{\pi} \int_{0}^{\infty} \lambda^{\alpha-1}(\lambda I+L)^{-1} L x d \lambda=L^{\alpha} x .
$$

Let $\beta$ be in $(0,1)$. Applying the above identity with $\alpha=1-\beta$ gives

$$
L^{-\beta} y=\frac{\sin (\alpha \pi)}{\pi} \int_{0}^{\infty} \lambda^{\alpha-1}(\lambda I+L)^{-1} y d \lambda
$$

for all $y$ in the range of $L$, i.e., all $y \in H^{0}$. Making the change of variable $t^{2}=\lambda^{-1}$ gives the first identity in (11). Breaking the first integral of (11) into two integrals on $(0,1)$ and $(1, \infty)$, respectively, and making the change of variables $t \rightarrow t^{-1}$ in the second gives the second expression of (11).

Remark 2.1. We note that (16) is equivalent to

$$
\left\|T_{1}(t) f\right\|_{0} \leq\left(c_{0} / t\right)^{2}\|f\| .
$$

Using this, (14) and (16), it readily follow that the integrals appearing in (11) are all well defined Bochner integrals.

Remark 2.2. It is possible to extend these results to obtain negative powers of coercive (non-symmetric) bilinear forms but this extension is beyond the scope of the present paper.

\section{Numerical Integration.}

The numerical approximation to the integrals appearing in (11) is based on quadrature approximations to the scalar integrals:

$$
\begin{aligned}
I(\lambda) & =\int_{0}^{\infty} t^{2 \beta-1}\left(1+t^{2} \lambda\right)^{-1} d t \\
& =\int_{0}^{1} t^{2 \beta-1}\left(1+t^{2} \lambda\right)^{-1} d t+\int_{0}^{1} t^{1-2 \beta}\left(t^{2}+\lambda\right)^{-1} d t=: I_{1}(\lambda)+I_{2}(\lambda) .
\end{aligned}
$$


In the next sections, we develop and analyze three quadratures.

For latter reference, we define

$$
f_{\lambda}(t) \equiv f_{\lambda}^{1}(t):=\left(1+\lambda t^{2}\right)^{-1}, \quad \text { and } \quad f_{\lambda}^{2}(t):=\left(t^{2}+\lambda\right)^{-1} .
$$

3.1. Rectangle quadrature rule on graded partitions. We start with $I_{1}(\lambda)$. We propose a graded partition of $[0,1]$ to cope with the singular behavior of $t^{2 \beta-1}$ coupled with a one point quadrature. For a given positive integer $N$, we set

(i) $t_{1, i}^{N}:=(i / N)^{\frac{1}{2 \beta}}$ if $2 \beta-1<0$.

(ii) $t_{1, i}^{N}:=i / N$ if $2 \beta-1 \geq 0$.

Case (i) above corresponds to refinement at $t=0$ and is necessary to deal with the singularity of the integrand at zero. We note that it is possible to use the node formula of Case (i) even when $2 \beta-1>0$. This results in larger spacing of the nodes near $t=0$ compared to the uniform case and appears to be unnecessary.

The quadrature formula approximating $I_{1}(\lambda)$ reads

$$
I_{1}^{N}(\lambda):=\sum_{i=0}^{N-1} f_{\lambda}^{1}\left(t_{1, i}^{N, *}\right) \int_{t_{1, i}^{N}}^{t_{1, i+1}^{N}} t^{2 \beta-1}=\sum_{i=0}^{N-1} \frac{\left(t_{1, i+1}^{N}\right)^{2 \beta}-\left(t_{1, i}^{N}\right)^{2 \beta}}{2 \beta} f_{\lambda}^{1}\left(t_{1, i}^{N, *}\right) .
$$

Here $t_{1, i}^{N, *}$ is any point in the interval $\left(t_{1, i}^{N}, t_{1, i+1}^{N}\right]$. In Case (i), the coefficient on the rightmost sum of (18) simplifies to $(2 \beta N)^{-1}$.

We proceed similarly for $I_{2}(\lambda)$. In this case, the partitioning is given by

(iii) $t_{2, i}^{N}:=(i / N)^{\frac{1}{2-2 \beta}}$ if $1-2 \beta<0$.

(iv) $t_{2, i}^{N}:=i / N$ if $1-2 \beta \geq 0$.

We define

$$
\begin{aligned}
I_{2}^{N}(\lambda): & =\sum_{i=0}^{N-1} f_{\lambda}^{2}\left(t_{2, i}^{N, *}\right) \int_{t_{2, i}^{N}}^{t_{2, i+1}^{N}} t^{1-2 \beta} \\
& =\sum_{i=0}^{N-1} \frac{\left(t_{2, i+1}^{N}\right)^{2-2 \beta}-\left(t_{2, i}^{N}\right)^{2-2 \beta}}{2-2 \beta} f_{\lambda}^{2}\left(t_{2, i}^{N, *}\right) .
\end{aligned}
$$

Now, $t_{2, i}^{N, *}$ is any point in the interval $\left(t_{2, i}^{N}, t_{2, i+1}^{N}\right]$.

Before estimating the quadrature error, we provide a bound on the ratio of two consecutive intervals.

Lemma 3.1 (Subdivision Regularity). Let $N>0$ and $t_{i}=(i / N)^{\theta}$ for $\theta>0$, $i=0, \ldots N$. Then, there exists constant $0<\rho(\theta)<\infty$ only depending on $\theta$ such that

$$
\max _{i=1, . ., N-1} \frac{t_{i+1}-t_{i}}{t_{i}-t_{i-1}} \leq \rho(\theta) .
$$

Proof. For $\theta=1$, the above fraction is identically 1 and hence we take $\rho(1)=1$. For $\theta \neq 1$, we consider the function

$$
g(t):=\frac{t^{\theta}-(t-1)^{\theta}}{(t-1)^{\theta}-(t-2)^{\theta}} .
$$

Note that $g(i+1)$ coincides with the fraction in (20). Moreover, a simple computation shows that $g^{\prime}(t)=0$ if and only if

$$
(t-1)^{1-\theta}=\frac{1}{2}\left(t^{1-\theta}+(t-2)^{1-\theta}\right) .
$$


The strict concavity of $\theta<1$ and strict convexity for $\theta>1$ of $t^{1-\theta}$ imply that $g^{\prime}(t)$ cannot be zero for any $t$ and so $g(t)$ is monotone. Hence we can take

$$
\rho(\theta):=\sup _{t \in[0, \infty]} g(t)=\left\{\begin{array}{lll}
g(2)=2^{\theta}-1 & \text { when } & \theta>1 \\
\lim _{t \rightarrow \infty} g(t)=1 & \text { when } & \theta \leq 1
\end{array}\right.
$$

which ends the proof.

We now provide an estimate on the quadrature error

$$
e(\lambda):=I_{1}(\lambda)-I_{1}^{N}(\lambda)+I_{2}(\lambda)-I_{2}^{N}(\lambda) .
$$

Lemma 3.2 (Quadrature Error). For any $0<\beta<1$ and $\lambda>0$, we have

$$
|e(\lambda)| \leq \frac{2+\rho\left(A^{-1}\right) \pi}{N A}+\frac{2+\rho\left(B^{-1}\right) \pi}{\lambda N B}
$$

where $\rho(\cdot)$ is given by (21). Here $A=2 \beta$ when we use (i) and $A=1$ when $2 \beta-1>0$ and we use (ii). Similarly, $B=2-2 \beta$ when we use (iii) and $B=1$ when $1-2 \beta>0$ and we use (iv).

Proof. Let us start by examining $e^{1}(\lambda):=I_{1}(\lambda)-I_{1}^{N}(\lambda)$. We first observe that when we use (i), the mean value theorem implies

$$
\begin{aligned}
\mid \int_{t_{1, i}^{N}}^{t_{1, i+1}^{N}} t^{2 \beta-1}\left(f_{\lambda}(t)\right. & \left.-f_{\lambda}\left(t_{1, i}^{N, *}\right)\right) d t \mid \\
& \leq \max _{s, t \in\left(t_{1, i}^{N}, t_{1, i+1}^{N}\right]}\left|f_{\lambda}(t)-f_{\lambda}(s)\right| \int_{t_{1, i}^{N}}^{t_{1, i+1}^{N}} t^{2 \beta-1} \\
& \leq \frac{1}{N A}\left(t_{1, i+1}^{N}-t_{1, i}^{N}\right)\left\|\left(f_{\lambda}\right)^{\prime}\right\|_{L_{\infty}\left(\left[t_{1, i}^{N}, t_{1, i+1}^{N}\right]\right) .}
\end{aligned}
$$

In contrast, if $2 \beta-1>0$ and we use (ii), the mean value theorem implies that

$$
\frac{\left(t_{1, i+1}^{N}\right)^{2 \beta}-\left(t_{1, i}^{N}\right)^{2 \beta}}{2 \beta} \leq \frac{\left(t_{1, i+1}^{N}\right)^{2 \beta-1}}{N} \leq \frac{1}{N} .
$$

and hence

$$
\begin{aligned}
\mid \int_{t_{1, i}^{N}}^{t_{1, i+1}^{N}} t^{2 \beta-1}\left(f_{\lambda}(t)\right. & \left.-f_{\lambda}\left(t_{1, i}^{N, *}\right)\right) d t \mid \\
& \leq \frac{\left(t_{1, i+1}^{N}\right)^{2 \beta}-\left(t_{1, i}^{N}\right)^{2 \beta}}{2 \beta} \max _{s, t \in\left(t_{1, i}^{N}, t_{1, i+1}^{N}\right]}\left|f_{\lambda}(t)-f_{\lambda}(s)\right| \\
& \leq \frac{1}{N A}\left(t_{1, i+1}^{N}-t_{1, i}^{N}\right)\left\|\left(f_{\lambda}\right)^{\prime}\right\|_{L_{\infty}\left(\left[t_{1, i}^{N}, t_{1, i+1}^{N}\right]\right)}
\end{aligned}
$$

We claim that for any $t>0$,

$$
\left|\left(f_{\lambda}\right)^{\prime}(t)\right|=\frac{2 \lambda t}{\left(1+\lambda t^{2}\right)^{2}} \leq \frac{2 \lambda^{1 / 2}}{1+\lambda t^{2}} .
$$

To see this, we distinguish two cases. If $\lambda^{1 / 2} t \leq 1$ then

$$
\left|\left(f_{\lambda}\right)^{\prime}(t)\right| \leq \frac{2 \lambda^{1 / 2}}{\left(1+\lambda t^{2}\right)^{2}} \leq \frac{2 \lambda^{1 / 2}}{1+\lambda t^{2}}
$$


Alternatively, if $\lambda^{1 / 2} t>1$ then

$$
\left|\left(f_{\lambda}\right)^{\prime}(t)\right| \leq 2 \lambda^{1 / 2} \frac{\lambda t^{2}}{\left(1+\lambda t^{2}\right)^{2}}=2 \lambda^{1 / 2}\left(\frac{1}{1+\lambda t^{2}}-\frac{1}{\left(1+\lambda t^{2}\right)^{2}}\right) \leq \frac{2 \lambda^{1 / 2}}{1+\lambda t^{2}} .
$$

Thus,

$$
\left\|\left(f_{\lambda}\right)^{\prime}\right\|_{L_{\infty}\left(\left[t_{1, i}^{N}, t_{1, i+1}^{N}\right]\right)} \leq \frac{2 \lambda^{1 / 2}}{1+\lambda\left(t_{1, i}^{N}\right)^{2}}
$$

It follows from (22), (24) and (25) that

$$
\left|e^{1}(\lambda)\right| \leq\left|e_{0}^{1}(\lambda)\right|+\frac{2}{N A} \sum_{i=1}^{N-1}\left(t_{1, i+1}^{N}-t_{1, i}^{N}\right) \frac{\lambda^{1 / 2}}{1+\lambda\left(t_{1, i}^{N}\right)^{2}} .
$$

where

$$
e_{0}^{1}(\lambda):=\int_{0}^{t_{1}} t^{2 \beta-1}\left(f_{\lambda}(t)-f_{\lambda}\left(t_{1,0}^{N, *}\right)\right) d t
$$

Setting $x_{i}=\lambda^{1 / 2} t_{1, i}^{N}$ gives

$$
\left|e^{1}(\lambda)\right| \leq\left|e_{0}^{1}(\lambda)\right|+\frac{2}{N A} \sum_{i=1}^{N-1} \frac{x_{i+1}-x_{i}}{1+x_{i}^{2}}
$$

By Lemma 3.1,

$$
\max _{i=1, . ., N-1} \frac{x_{i+1}-x_{i}}{x_{i}-x_{i-1}}=\max _{i=1, . ., N-1} \frac{t_{i+1}-t_{i}}{t_{i}-t_{i-1}} \leq \rho\left(A^{-1}\right) .
$$

Thus,

$$
\begin{aligned}
\left|e^{1}(\lambda)\right| & \leq\left|e_{0}^{1}(\lambda)\right|+\frac{2 \rho\left(A^{-1}\right)}{N A} \sum_{i=1}^{N-1} \frac{x_{i}-x_{i-1}}{1+x_{i}^{2}} \\
& \leq\left|e_{0}^{1}(\lambda)\right|+\frac{2 \rho\left(A^{-1}\right)}{N A} \int_{0}^{\infty} \frac{1}{1+x^{2}} d x \\
& =\left|e_{0}^{1}(\lambda)\right|+\frac{2 \rho\left(A^{-1}\right)}{N A} \pi
\end{aligned}
$$

where the second inequality followed from the monotone decreasing property of $1 /\left(1+x^{2}\right)$.

The error on the first interval is bounded by

$$
\begin{aligned}
\left|e_{0}^{1}(\lambda)\right| & \leq \int_{0}^{t_{1,1}^{N}} t^{2 \beta-1}\left(\left|f_{\lambda}(t)\right|+\left|f_{\lambda}\left(t_{1,0}^{N, *}\right)\right|\right) d t \\
& \leq 2 \int_{0}^{t_{1,1}^{N}} t^{2 \beta-1} \leq \frac{2}{N A} .
\end{aligned}
$$

The last inequality followed from (23) when $2 \beta-1>0$ and we use (ii) and is an obvious equality in Case (i).

Gathering the above estimates, we arrive at

$$
\left|e^{1}(\lambda)\right| \leq \frac{2+2 \rho\left(A^{-1}\right) \pi}{N A}
$$

To bound $e^{2}(\lambda):=I_{2}(\lambda)-I_{2}^{N}(\lambda)$, we first observe that

$$
I_{2}(\lambda)=\lambda^{-1} \int_{0}^{1} t^{1-2 \beta} f_{\lambda^{-1}}(t) d t .
$$


and

$$
I_{2}^{N}(\lambda)=\lambda^{-1} \sum_{i=0}^{N-1} f_{\lambda^{-1}}\left(t_{2, i}^{N, *}\right) \int_{t_{2, i}^{N}}^{t_{2, i+1}^{N}} t^{1-2 \beta} d t .
$$

We see that the quadratures $I_{1}^{N}$ and $I_{2}^{N}$ are similarly constructed only differing in that the partitioning is tailored to the power of $t$ in the approximated integral. All of the above arguments apply to $\lambda\left(I_{2}-I_{2}^{N}\right)$ and lead to

$$
\lambda\left|e^{2}(\lambda)\right| \leq \frac{2+2 \rho\left(B^{-1}\right) \pi}{N B} .
$$

Combining (27) and (28) completes the proof of the lemma.

To illustrate the sharpness of the quadrature error estimate (Lemma 3.2), we (approximately) compute $\|e(\lambda)\|_{L^{\infty}(10, \infty)}$ by the following algorithm:

(I) We examine the value of $\left|e\left(\lambda_{i}\right)\right|$ for $\lambda_{i}=10 \mu^{i}$ for $i=0,1, \ldots M$. Here $\mu>1$ and $M$ is chosen so large that $\left|e\left(\lambda_{i}\right)\right|$ appears monotonically decreasing for larger $i$. In any event, $\lambda_{M}$ is on the order of $10^{7}$ or larger, a value which is well beyond our range of applications.

(II) We then refine in a neighborhood of the $\lambda_{j}$ which obtains the maximum of $\left|e\left(\lambda_{i}\right)\right|, i=0, \ldots, M$ and select the maximizing $\lambda$ from the refined values.

Proving the convergence of such a procedure is beyond the scope of this paper however the plots of $\left|e\left(\lambda_{i}\right)\right|$ appear to be sufficiently well behaved and suggest that the algorithm works.

The values of $\|e(\lambda)\|_{L^{\infty}(10, \infty)}$ (as computed above) are reported in Table 1 as a function of $\beta=1 / 4,1 / 2,3 / 4$ and $N$. For these runs, we chose $t_{j, k}^{N \text {,* }}$ to be the node corresponding to a one point weighted Gaussian quadrature. Specifically,

$$
Q_{1, k}(f)=\left(t_{1, k+1}^{N}-t_{1, k+1}^{N}\right) f\left(t_{1, k+1}^{N, *}\right) \approx \int_{t_{1, k+1}^{N}}^{t_{1, k+1}^{N}} t^{1-2 \beta} f d x
$$

and

$$
Q_{2, k}(f)=\left(t_{2, k+1}^{N}-t_{2, k+1}^{N}\right) f\left(t_{2, k+1}^{N, *}\right) \approx \int_{t_{2, k+1}^{N}}^{t_{2, k+1}^{N}} t^{2 \beta-1} f d x
$$

with $t_{j, k}^{N, *}$ chosen to make the schemes exact for cubics.

In the case of $\beta=.5$, there is no distinction between the cases (i)-(ii) and (iii)(iv) since all result in equally spaced meshes. When $\beta=.25$, we used (i) and (iv) for the respective integrals. We note, however, that switching from (iv) to (iii) in this case made negligible difference in the observed maximum errors. For $\beta=.75$, the first column reports (ii)-(iii). In this case, switching to (i)-(iii) leads to a slower convergent first order method.

3.2. Gaussian quadrature rule on geometric partitioning. We start with the numerical integration of $I_{1}(\lambda)$. In contrast with the graded subdivision used in Section 3.1 , we propose this time a geometrically refined partition of $[0,1]$ to cope with the singular behavior of $t^{2 \beta-1}$.

For a given positive integer $M$ yet to be chosen, we consider the dyadic partition $[0,1]=\cup_{i=1}^{M} I_{i} \cup\left[0,2^{-M}\right]$, where

$$
I_{i}=\left[2^{-i}, 2^{-i+1}\right], \quad i=1, \ldots, M .
$$




\begin{tabular}{|c|c|c|c|}
\hline$N$ & $\beta=.5$ & $\beta=.75$ & $\beta=.25$ \\
\hline 31 & $2.86 \times 10^{-3}$ & $1.60 \times 10^{-4}$ & $7.77 \times 10^{-3}$ \\
\hline 63 & $1.40 \times 10^{-3}$ & $5.51 \times 10^{-5}$ & $3.82 \times 10^{-3}$ \\
\hline 127 & $6.98 \times 10^{-4}$ & $1.93 \times 10^{-5}$ & $1.89 \times 10^{-3}$ \\
\hline 255 & $3.45 \times 10^{-4}$ & $6.75 \times 10^{-6}$ & $9.45 \times 10^{-4}$ \\
\hline 511 & $1.73 \times 10^{-4}$ & $2.39 \times 10^{-6}$ & $4.71 \times 10^{-4}$ \\
\hline 1023 & $8.66 \times 10^{-5}$ & $8.38 \times 10^{-7}$ & $2.35 \times 10^{-4}$ \\
\hline
\end{tabular}

TABLE 1. $\|e(\lambda)\|_{L^{\infty}(10, \infty)}$ for different $\beta$ (rectangular scheme). The observed order is $N^{-1}(\beta=0.5$ and $\beta=0.25)$ and $N^{-3 / 2}$ $(\beta=0.75)$.

Furthermore, given an integer $N$, each of these intervals $I_{i}$ are decomposed onto $N$ uniform subintervals $I_{i, j}, j=1, \ldots, N$, of length

$$
\left|I_{i, j}\right|=\frac{1}{2^{i} N}, \quad i=1, \ldots, M, \quad j=1, \ldots, N .
$$

Given an integer $r \geq 1$, a $r$ points weighted Gaussian quadrature with weight $w(t)=t^{2 \beta-1}$ is applied on each intervals $I_{i, j}$. We denote by $t_{i, j}^{1, k}$ and by $A_{i, j}^{1, k}$, $1 \leq i \leq M, 1 \leq j \leq N$ and $1 \leq k \leq r$ the Gaussian quadrature points and weights respectively. Hence, the quadrature formula on $I_{i, j}$ reads

$$
I_{i, j}^{N, r}(\lambda):=\sum_{k=1}^{r} f_{\lambda}^{1}\left(t_{i, j}^{1, k}\right) A_{i, j}^{1, k}
$$

and we propose the following expression for the approximation of $I_{1}(\lambda)$ :

$$
I_{1}(\lambda) \approx I_{1}^{N, r}(\lambda):=\sum_{i=1}^{M} \sum_{j=1}^{N} I_{i, j}^{N, r}(\lambda) .
$$

Notice that we dropped the contribution of the first interval $\left[0,2^{-M}\right]$. The quadrature error $e_{1}(\lambda):=I_{1}(\lambda)-I_{1}^{N, r}(\lambda)$ is given by

$$
e_{1}(\lambda)=\sum_{i=1}^{M} \sum_{j=1}^{N} e_{i, j}^{1}+\int_{0}^{2^{-M}} t^{2 \beta-1} f_{\lambda}^{1}(t) d t
$$

where $e_{i, j}^{1}$ are the quadrature errors over each subintervals $I_{i, j}$ defined as

$$
e_{i, j}^{1}(\lambda):=\int_{I_{i, j}} t^{2 \beta-1} f_{\lambda}^{1}(t) d t-\sum_{k=1}^{r} f_{\lambda}^{1}\left(t_{i, j}^{k}\right) A_{i, j}^{k} .
$$

At this point, it is worth recalling that for some $\xi_{i, j} \in I_{i, j}$, we have the following expression for the local quadrature errors

$$
e_{i, j}^{1}(\lambda)=\frac{1}{(2 r) !} \frac{d^{2 r}}{d t^{2 r}} f_{\lambda}^{1}\left(\xi_{i, j}\right) \int_{I_{i, j}} t^{2 \beta-1}\left(q_{i, j}^{1}\right)^{2}(t) d t,
$$

where $q_{i, j}^{1}(t)=\prod_{k=1}^{r}\left(t-t_{i, j}^{1, k}\right)$. 
We proceed similarly for $I_{2}(\lambda)$. In this case, we set $w(t):=t^{1-2 \beta}$ so that the quadrature error for the second integral $I_{2}(\lambda)$ reads

$$
e^{2}(\lambda)=\sum_{i=1}^{M} \sum_{j=1}^{N} e_{i, j}^{2}+\int_{0}^{2^{-M}} t^{1-2 \beta} f_{\lambda}^{2}(t) d t
$$

where

$$
e_{i, j}^{2}(\lambda):=\int_{I_{i, j}} t^{1-2 \beta} f_{\lambda}^{2}(t) d t-\sum_{k=1}^{r} f_{\lambda}^{2}\left(t_{i, j}^{2, k}\right) A_{i, j}^{2, k} .
$$

Here $t_{i, j}^{2, k}$ and $A_{i, j}^{2, k}$ are the Gaussian points and weights. Again, the local quadrature error $e_{i, j}^{2}(\lambda)$ satisfies

$$
e_{i, j}^{2}(\lambda)=\frac{1}{(2 r) !} \frac{d^{2 r}}{d t^{2 r}} f_{\lambda}^{2}\left(\xi_{i, j}\right) \int_{I_{i, j}} t^{1-2 \beta}\left(q_{i, j}^{2}\right)^{2}(t) d t
$$

for some $\xi_{i, j} \in I_{i, j}$ and where $q_{i, j}^{2}(t):=\prod_{k=1}^{r}\left(t-t_{i, j}^{2, k}\right)$.

Notice that the dependency in the number of geometric intervals $M$ is never specified in the above defined quantities. In fact, we are now in position to provide its value

$$
M:=\left\lceil\frac{r \log _{2}(N)}{\min (\beta, 1-\beta)}\right\rceil .
$$

A slightly better algorithm is obtained by using a different number of geometric intervals for each integral, i.e.,

$$
M_{1}:=\left\lceil\frac{r \log _{2}(N)}{\beta}\right\rceil \text { and } \quad M_{2}:=\left\lceil\frac{r \log _{2}(N)}{1-\beta}\right\rceil .
$$

As we shall see in the proof of Lemma 3.3, this choice guarantee that contribution

$$
\int_{0}^{2^{-M}} t^{2 \beta-1} f_{\lambda}^{1}(t) d t \text { and } \int_{0}^{2^{-M}} t^{1-2 \beta} f_{\lambda}^{2}(t) d t
$$

present in (30) and (33) decays optimally. More precisely, we show that the quadrature error

$$
e(\lambda):=e_{1}(\lambda)+e_{2}(\lambda)
$$

decays like $N^{-2 r}$ (independently of $\lambda \geq \lambda_{0}$ ) when using $r M$ quadrature points per integral as above.

Lemma 3.3 (Quadrature Error). There exists a constant $C_{G}$ only depending on $r$ such that the quadrature error $e(\lambda)$ using $\left\lceil\frac{r^{2} N \log _{2} N}{\min (\beta, 1-\beta)}\right\rceil$ quadrature points satisfies

$$
e(\lambda) \leq C_{G}\left(\frac{1}{2 \beta}+\frac{1}{2(1-\beta) \lambda}\right) N^{-2 r} .
$$

Proof. We start with the quadrature approximation of $I_{1}(\lambda)$ and focus on the subinterval $I_{i, j}$ for some $1 \leq i \leq M$ and $1 \leq j \leq N$. Owing to the error representation formula (32) and the relation (29) providing the length of $I_{i, j}$, we directly obtain that

$$
e_{i, j}^{1}(\lambda) \leq \frac{1}{(2 r) !}\left|\frac{d^{2 r}}{d t^{2 r}} f_{\lambda}^{1}\left(\xi_{i, j}\right)\right|\left(\frac{1}{2^{i}}\right)^{2 r} N^{-2 r} \int_{I_{i, j}} t^{2 \beta-1} d t
$$


We compute

$$
\frac{d^{2 r}}{d t^{2 r}} f_{\lambda}^{1}(t)=\sum_{r \leq k \leq 2 r} c_{k} \frac{\lambda^{k} t^{2 k-2 r}}{\left(1+t^{2} \lambda\right)^{1+k}}
$$

where $c_{k}$ are absolute constants. Therefore, $\xi_{i, j} \in I_{i, j}$ implies

$\frac{d^{2 r}}{d t^{2 r}} f_{\lambda}^{1}\left(\xi_{i, j}\right)\left(\frac{1}{2^{i}}\right)^{2 r} \leq 2^{2 r} \frac{d^{2 r}}{d t^{2 r}} f_{\lambda}^{1}\left(\xi_{i, j}\right) \xi_{i, j}^{2 r} \leq 2^{2 r} \sum_{r \leq k \leq 2 r}\left|c_{k}\right| \frac{\lambda^{k} \xi_{i, j}^{2 k}}{\left(1+\xi_{i, j}^{2} \lambda\right)^{1+k}} \leq 2^{2 r} \sum_{r \leq k \leq 2 r}\left|c_{k}\right|$.

Hence, setting $C=\frac{2^{2 r}}{(2 r) !} \sum_{r \leq k \leq 2 r}\left|c_{k}\right|$,

$$
e_{i, j}^{1}(\lambda) \leq C N^{-2 r} \int_{I_{i, j}} t^{2 \beta-1}
$$

so that

$$
\sum_{i=1}^{M} \sum_{j=1}^{N} e_{i, j}^{1}(\lambda) \leq C N^{-2 r} \int_{2^{-M}}^{1} t^{2 \beta-1} \leq \frac{C}{2 \beta} N^{-2 r}
$$

In view of $(30)$, it remains to bound $\int_{0}^{2^{-M}} t^{2 \beta-1} f_{\lambda}^{1}(t) d t$. Notice that $f_{\lambda}^{1}(t) \leq 1$ yields

$$
\int_{0}^{2^{-M}} t^{2 \beta-1} f_{\lambda}^{1}(t) d t \leq \frac{1}{2 \beta} 2^{-2 \beta M} \leq \frac{1}{2 \beta} N^{-2 r},
$$

where we used the definition (35) of $M$ for the last inequality. The estimate for $e^{1}(\lambda)$ follows upon gathering the last two estimates.

We now discuss the approximation of $I_{2}(\lambda)$. In this case, notice that there holds

$$
\frac{d^{2 r}}{d t^{2 r}} f_{\lambda}^{2}(t)=\sum_{r \leq k \leq 2 r} \tilde{c}_{k} \frac{t^{2 k-2 r}}{\left(t^{2}+\lambda\right)^{1+k}}
$$

where $\tilde{c}_{k}$ are absolute constants. As a consequence,

$$
\frac{d^{2 r}}{d t^{2 r}} f_{\lambda}^{2}\left(\xi_{i, j}\right) \xi_{i, j}^{2 r} \leq \sum_{r \leq k \leq 2 r} \frac{1}{\lambda}\left|\tilde{c}_{k}\right|
$$

and we directly obtain

$$
\sum_{i=1}^{M} \sum_{j=1}^{N} e_{i, j}^{1}(\lambda) \leq \frac{\tilde{C}}{2(1-\beta) \lambda} N^{-2 r}
$$

where $\tilde{C}=\frac{2^{2 r}}{(2 r) !} \sum_{r \leq k \leq 2 r}\left|\tilde{c}_{k}\right|$. The contribution from the first interval follows noticing that $f_{\lambda}^{2}(t) \leq \frac{1}{\lambda}$ and as in the first case,

$$
\int_{0}^{2^{-M}} t^{1-2 \beta} f_{\lambda}^{2}(t) d t \leq \frac{1}{\lambda} \frac{2^{2(1-\beta) M}}{2(1-\beta)} \leq \frac{1}{\lambda} \frac{N^{-r}}{2(1-\beta)} .
$$

This ends the estimate for $e^{2}(\lambda)$ and the proof.

To illustrate numerically the performance of our quadrature scheme. We proceed as in Section 3.1 and compare

with

$$
\|e(\lambda)\|_{L_{\infty}(10, \infty)}
$$

$N S Y S:=$ number of system solves. 
The number of geometric intervals for each of the two integrals was determined by (36). Table 2 reports the values of $\|e(\lambda)\|_{L_{\infty}(10, \infty)}$ when using 2 Gaussian points (i.e. $r=2$ ) for different values of $N$. Except for the case of $N=2$ and $\beta=.75$, the errors for the geometric scheme were smaller than those of the rectangle quadrature rule.

\begin{tabular}{|c|c|c|c|}
\hline$N$ & $\beta=.5(N S Y S)$ & $\beta=.75(N S Y S)$ & $\beta=.25(N S Y S)$ \\
\hline 2 & $1.37 \times 10^{-3}(72)$ & $8.37 \times 10^{-4}(92)$ & $2.55 \times 10^{-3}(92)$ \\
\hline 4 & $8.58 \times 10^{-5}(208)$ & $4.16 \times 10^{-5}(272)$ & $1.58 \times 10^{-4}(272)$ \\
\hline 8 & $5.36 \times 10^{-6}(544)$ & $4.22 \times 10^{-6}(704)$ & $1.00 \times 10^{-5}(704)$ \\
\hline 16 & $3.35 \times 10^{-7}(1344)$ & $2.05 \times 10^{-7}(1760)$ & $6.22 \times 10^{-7}(1760)$ \\
\hline
\end{tabular}

TABLE 2. Two points Gaussian quadrature based on a dyadic partition. The observed order of convergence is $N^{-4}$ as predicted by Lemma 3.3.

3.3. An exponentially convergent quadrature. We next introduce a quadrature scheme which provides exponential convergence to (5), specifically,

$$
\mathcal{Q}^{\beta}=\frac{2 k \sin (\pi \beta)}{\pi} \sum_{\ell=-N}^{N} e^{2 \beta y_{\ell}}\left(I+e^{2 y \ell} L\right)^{-1}
$$

with $y_{\ell}=\ell k$ and $k=1 / \sqrt{N}$.

Let $\mathcal{Q}(\lambda)$ for $\lambda \geq \lambda_{0}>0$ be the expression on the right hand side of (37) with $L$ replaced by $\lambda$. We apply the classical analysis for these types of quadrature approximations given in [24], specifically, Theorem 2.20 of [24]. Estimates for $\left\|L^{-\beta}-\mathcal{Q}^{\beta}\right\|$ will follow from estimates for $\left|\lambda^{-\beta}-\mathcal{Q}^{\beta}(\lambda)\right|$ which are uniform for $\lambda \geq \lambda_{0}$.

We first note that for $\eta \in \mathbb{R},|\eta| \leq \pi / 4$ and $\lambda \geq \lambda_{0}$,

$$
\left|\left(1+e^{2(y+i \eta)} \lambda\right)^{-1}\right| \leq\left\{\begin{aligned}
1: & \text { for } y \leq 0, \\
e^{-2 y} \lambda_{0}^{-1}: & \text { for } y>0 .
\end{aligned}\right.
$$

It easily follows that $g_{\lambda}(z):=e^{2 \beta z}\left(1+e^{2 z} \lambda\right)^{-1}$ is analytic in the strip $D_{S}=\{z \in$ $\mathbb{C},|\Im(z)|<\pi / 4\}$ for every $\lambda>0$. In addition, (38) implies that for $|\eta| \leq \pi / 4$ and $\lambda \geq \lambda_{0}$,

$$
\left|g_{\lambda}(y+i \eta)\right| \leq\left\{\begin{aligned}
e^{2 \beta y}: & \text { for } y \leq 0, \\
e^{-(2-2 \beta) y} \lambda_{0}^{-1}: & \text { for } y>0 .
\end{aligned}\right.
$$

This implies that

$$
\begin{aligned}
N\left(D_{S}\right) & :=\max _{\lambda \geq \lambda_{0}}\left\{\int_{-\infty}^{\infty}\left(\left|g_{\lambda}(y-i \pi / 4)\right|+\left|g_{\lambda}(y+i \pi / 4)\right|\right) d y\right\} \\
& \leq \beta^{-1}+\left((1-\beta) \lambda_{0}\right)^{-1} .
\end{aligned}
$$

The above considerations readily imply that $g_{\lambda}$ is in $B\left(D_{S}\right)$ for $\lambda \geq \lambda_{0}$, where $B\left(D_{S}\right)$ is the set of functions analytic in $D_{S}$ that satisfy

$$
\int_{-\pi / 4}^{\pi / 4}\left|g_{\lambda}(t+i y)\right| d y=\mathcal{O}\left(|t|^{\alpha}\right), \quad t \rightarrow \pm \infty, \quad 0 \leq a<1,
$$


(see Definition 2.12 of [24]). We can apply Theorem 2.20 of [24] to conclude that for $k>0$,

$$
\left|\int_{-\infty}^{\infty} g_{\lambda}(y) d y-k \sum_{\ell=-\infty}^{\infty} g_{\lambda}(\ell k)\right| \leq \frac{N\left(D_{S}\right)}{2 \sinh \left(\pi^{2} /(4 k)\right)} e^{-\pi^{2} /(4 k)} .
$$

The following lemma is an immediate consequence of (41) and the obvious estimates,

$$
\begin{aligned}
& k \sum_{\ell=-N-1}^{-\infty}\left|g_{\lambda}(\ell k)\right| \leq \frac{1}{2 \beta} e^{-2 \beta / k} \\
& k \sum_{\ell=N+1}^{\infty}\left|g_{\lambda}(\ell k)\right| \leq \frac{1}{(2-2 \beta) \lambda_{0}} e^{-(2-2 \beta) / k}
\end{aligned}
$$

Lemma 3.4 (Quadrature Error). Let $\mathcal{Q}^{\beta}(\lambda)$ be defined by (37) with L replaced by $\lambda \geq \lambda_{0}, N>0$ and $k=1 / \sqrt{N}$. Then

$$
\begin{aligned}
\left|\lambda^{-\beta}-\mathcal{Q}^{\beta}(\lambda)\right| \leq & \frac{2 \sin (\pi \beta)}{\pi}\left[\frac{N\left(D_{S}\right)}{2 \sinh \left(\pi^{2} /(4 k)\right)} e^{-\pi^{2} /(4 k)}\right. \\
& \left.+\frac{1}{2 \beta} e^{-2 \beta / k}+\frac{1}{(2-2 \beta) \lambda_{0}} e^{-(2-2 \beta) / k}\right] .
\end{aligned}
$$

Remark 3.1 (Exponential Decay). The error from the three exponentials above can essentially be equalized by setting

$$
\mathcal{Q}^{\beta}(\lambda)=\frac{2 k \sin (\pi \beta)}{\pi} \sum_{\ell=-M}^{N} g_{\lambda}(\ell k)
$$

with

$$
\pi^{2} /(2 k) \approx 2 \beta k M \approx(2-2 \beta) k N .
$$

Thus, given $k>0$, we set

$$
M=\left\lceil\frac{\pi^{2}}{4 \beta k^{2}}\right\rceil \quad \text { and } \quad N=\left\lceil\frac{\pi^{2}}{4(1-\beta) k^{2}}\right\rceil
$$

and get the estimate

$$
\left|\lambda^{-\beta}-\mathcal{Q}^{\beta}(\lambda)\right| \leq \frac{2 \sin (\pi \beta)}{\pi}\left[\frac{1}{2 \beta}+\frac{1}{(2-2 \beta) \lambda_{0}}\right]\left[\frac{e^{-\pi^{2} /(4 k)}}{\sinh \left(\pi^{2} /(4 k)\right)}+e^{-\pi^{2} /(2 k)}\right] .
$$

We note that the right hand side above asymptotically behaves like

$$
\frac{2 \sin (\pi \beta)}{\pi}\left(\frac{1}{\beta}+\frac{1}{(1-\beta) \lambda_{0}}\right) e^{-\pi^{2} /(2 k)}
$$

as $k \rightarrow 0$.

The convergence results for the exponential scheme are illustrated in Table 3 . As usual, we report the total number of systems needed to be solved ( $N S Y S)$. This scheme produces errors (using a comparable number of iterations) which are smaller than those of the other two schemes. We use the equalized exponential scheme of the previous remark. 


\begin{tabular}{|c|c|c|c|}
\hline$k$ & $\beta=.5(N S Y S)$ & $\beta=.75(N S Y S)$ & $\beta=.25(N S Y S)$ \\
\hline 1 & $2.71 \times 10^{-3}(11)$ & $7.62 \times 10^{-4}(15)$ & $4.77 \times 10^{-3}(15)$ \\
\hline $1 / 2$ & $2.45 \times 10^{-5}(41)$ & $9.15 \times 10^{-6}(55)$ & $3.65 \times 10^{-5}(55)$ \\
\hline $1 / 3$ & $1.80 \times 10^{-7}(91)$ & $1.01 \times 10^{-7}(120)$ & $3.06 \times 10^{-7}(120)$ \\
\hline $1 / 4$ & $1.63 \times 10^{-9}(159)$ & $8.01 \times 10^{-10}(212)$ & $2.29 \times 10^{-9}(212)$ \\
\hline
\end{tabular}

TABle 3. Error for the exponential scheme with $N$ and $M$ as in Remark 3.1.

3.4. Operator approximation, the finite dimensional case. We use the quadrature approximations of the previous section to develop approximations to the integrals of Theorem 2.1 in the finite dimensional case. Let $H$ be a finite dimensional space of dimension $K$ and set $H^{1}=H^{0}=H$. We assume that we have two inner products, $\langle\cdot, \cdot\rangle_{i}, i=1,2$, defined on $H$ satisfying (10).

The quadrature described in Sections 3.1, 3.2 and 3.3 yield three different approximations of the $T^{\beta}$ :

$$
\begin{aligned}
& \mathcal{Q}_{R}^{\beta}:=\frac{1}{2 N C_{\beta}} \sum_{j=1}^{N}\left[\beta^{-1} T_{1}\left(t_{1, j}^{N, *}\right)+(1-\beta)^{-1} T_{2}\left(t_{2, j}^{N, *}\right)\right], \\
& \mathcal{Q}_{G}^{\beta}:=\frac{1}{C_{\beta}} \sum_{i=1}^{M} \sum_{j=1}^{N} \sum_{k=1}^{r}\left(A_{i, j}^{1, k} T_{1}\left(t_{i, j}^{1, k}\right)+A_{i, j}^{2, k} T_{2}\left(t_{i, j}^{2, k}\right)\right), \quad \text { and } \\
& \mathcal{Q}_{E}^{\beta}:=\frac{k}{C_{\beta}} \sum_{i=-N}^{N} e^{2 \beta y_{\ell}} T_{1}\left(e^{2 y_{\ell}}\right), \quad y_{\ell}=\ell k, \quad k=1 / \sqrt{N} .
\end{aligned}
$$

We use Lemmas 3.2, 3.3 and 3.4 to obtain:

Theorem 3.5. Let $H^{1}=H^{0}=H$ with $H$ finite dimensional and $\mathcal{Q}_{R}^{\beta}, \mathcal{Q}_{G}^{\beta}$ and $\mathcal{Q}_{E}^{\beta}$ be defined by (42), (43) and (44), respectively. Then

$$
\begin{aligned}
& \left\|T^{\beta}-\mathcal{Q}_{R}^{\beta}\right\|_{0} \leq B_{R}:=\frac{2+\rho\left((2 \beta)^{-1}\right) \pi}{2 C_{\beta} N \beta}+\frac{\mu\left(2+\rho\left((2-2 \beta)^{-1}\right) \pi\right)}{2 C_{\beta} N(1-\beta)}, \\
& \left\|T^{\beta}-\mathcal{Q}_{G}^{\beta}\right\|_{0} \leq B_{G}:=\frac{C_{G}}{C_{\beta}}\left(\frac{1}{2 \beta}+\frac{\mu}{2(1-\beta)}\right) N^{-2 r}
\end{aligned}
$$

and

$$
\begin{aligned}
\left\|T^{\beta}-\mathcal{Q}_{E}^{\beta}\right\|_{0} \leq B_{E}:=\frac{1}{C_{\beta}} & {\left[\frac{N\left(D_{S}\right)}{2 \sinh \left(\pi^{2} /(4 k)\right)} e^{-\pi^{2} /(4 k)}\right.} \\
& \left.+\frac{1}{2 \beta} e^{-2 \beta / k}+\frac{\mu}{(2-2 \beta)} e^{-(2-2 \beta) / k}\right] .
\end{aligned}
$$

Here $\mu$ denotes the largest eigenvalue of $T, C_{G}$ denotes the constant only depending on $r$ appearing in Lemma 3.3 and $k=1 / \sqrt{N}$.

Proof. We let $\mathcal{Q}^{\beta}$ denote either $\mathcal{Q}_{R}^{\beta}, \mathcal{Q}_{G}^{\beta}$ or $\mathcal{Q}_{E}^{\beta}$. As in Section 2, we use the $\langle\cdot, \cdot\rangle_{0}$ orthogonal basis of eigenfunctions $\left\{\widetilde{\psi}_{i}\right\}, i=1, \ldots, K$ and their non-increasing eigenvalues $\left\{\mu_{i}\right\}$. Expanding $f \in H$ as

$$
f=\sum_{i=1}^{N}\left\langle f, \widetilde{\psi}_{i}\right\rangle_{0} \widetilde{\psi}_{i},
$$


we find that

$$
\left(T^{\beta}-\mathcal{Q}^{\beta}\right) f=C_{\beta}^{-1} \sum_{i=1}^{N} e\left(\mu_{i}^{-1}\right)\left\langle f, \widetilde{\psi}_{i}\right\rangle_{0} \widetilde{\psi}_{i}
$$

where

$$
e(\lambda):=\lambda^{-\beta}-\mathcal{Q}^{\beta}(\lambda) .
$$

The theorem follows immediately from Lemmas 3.2, 3.3 and 3.4.

\section{Application to an elliptic boundary value problem}

We restrict our considerations to $H^{0}=L_{2}(\Omega)$ and $H^{1}=H_{0}^{1}(\Omega)$ with $\Omega$ a bounded polygonal domain in $\mathbb{R}^{d}, d=1,2,3$ with a Lipschitz continuous boundary. We denote by $\|\cdot\|_{L_{2}}$ and $\|\cdot\|_{H^{1}}$ the standard $L_{2}$ and $H^{1}$ norms respectively. In order to simplify the notations, we write $g_{1}(\cdot) \preceq g_{2}(\cdot)$ when $g_{1}(\cdot) \leq C g_{2}(\cdot)$ for a constant $C$ independent of the argument in $g_{1}$ and $g_{2}$.

Let $A(\cdot, \cdot)$ denote an $H^{1}$ coercive, symmetric bilinear form on $H^{1} \times H^{1}$, e.g.,

$$
A(v, w)=\int_{\Omega} a(x) \nabla v \cdot \nabla w d x
$$

with $a(x)$ satisfying $0<a_{0} \leq a(x) \leq a_{1}$ for positive numbers $a_{0}, a_{1}$. We take

$$
<v, w>_{1}=A(v, w) \quad \text { and } \quad<v, w>_{0}=\int_{\Omega} v w d x .
$$

Clearly, Condition (a) of Section 2 holds for this pair of spaces. Moreover, Condition (b) is just the Poincaré inequality which holds as well.

Let $H_{h} \subset H^{1}$ be the space of continuous piecewise linear finite element functions defined with respect to a quasi uniform triangulation $\mathcal{T}_{h}$ of $\Omega$. By this we mean that there exists $\rho>0$ independent of $h$ such that for any $h>0$

$$
\max _{T \in \mathcal{T}_{h}} \operatorname{diam}(T) \leq \rho \min _{T \in \mathcal{T}_{h}} \operatorname{diam}(T) .
$$

For $F \in H^{-1}(\Omega)$, the dual of $H^{1}=H_{0}^{1}(\Omega)$, the finite element approximation $T_{h}(F)$ of $T(F)$ is defined by $T_{h}(F)=u_{h} \in H_{h}$ satisfying

$$
<u_{h}, \phi_{h}>_{1}=<F, \phi_{h}>, \quad \text { for all } \phi_{h} \in H_{h} .
$$

We shall approximate first $T^{\beta}$ by $T_{h}^{\beta} \pi_{h}$ and subsequently apply the results of Section 3.4 to develop an approximation to $T_{h}^{\beta} \pi_{h}$. Let $T_{1, h}$ be defined by (13) with $H^{1}$ replaced by $H_{h}$ and $T_{2, h}(t)=t^{-2} T_{1, h}\left(t^{-1}\right)$. Note that the definitions of $T_{i, h}(t) F, i=1,2$ make perfect sense for $F \in H^{-1}$. In addition, for $f \in H^{0}$,

$$
T_{i, h}(t) \pi_{h} f=T_{i, h}(t) f, \quad \text { for } i=1,2 .
$$

In addition, the assumption $H_{h} \subset H^{1}$ implies that (14) and (16) also hold for $T_{1, h}(t)$ and $T_{2, h}(t)$. We define $\mathcal{Q}_{R, h}^{\beta}, \mathcal{Q}_{G, h}^{\beta}$ and $\mathcal{Q}_{E, h}^{\beta}$ by replacing $T_{1}$ and $T_{2}$ by $T_{1, h}$ and $T_{2, h}$ in (42), (43) and (44).

The goal of this section is to estimate $\left\|\left(T^{\beta}-\mathcal{Q}_{h}^{\beta} \pi_{h}\right) f\right\|_{L_{2}}$ for appropriate $f$. Theorem 3.5 immediately leads to a bound for $\left\|\left(T_{h}^{\beta}-\mathcal{Q}_{h}^{\beta}\right) \pi_{h}\right\|_{L_{2}}$ and hence we need only estimate $\left\|\left(T^{\beta}-T_{h}^{\beta} \pi_{h}\right) f\right\|_{L_{2}}$. This, in turn, will require more precise 
regularity estimates for the operator $T$. To this end, we define intermediate spaces for any $-1 \leq s \leq 2$,

$$
\widetilde{H}^{s}(\Omega):= \begin{cases}H_{0}^{1}(\Omega) \cap H^{s}(\Omega), & 1 \leq s \leq 2 \\ {\left[L_{2}(\Omega), H_{0}^{1}(\Omega)\right]_{s},} & 0 \leq s \leq 1 \\ {\left[H^{-1}(\Omega), L_{2}\right]_{1+s},} & -1 \leq s \leq 0\end{cases}
$$

where $[\cdot, \cdot]_{s}$ denotes the real interpolation method and $H^{-1}(\Omega)=H^{-1}$ denotes the dual of $H_{0}^{1}(\Omega)$.

As already observed in Section 2, $T: H^{1} \rightarrow H^{-1}$ is an isomorphism, i.e., $T$ : $\widetilde{H}^{1}(\Omega) \rightarrow \widetilde{H}^{-1}(\Omega)$ is an isomorphism. For $s \geq-1$, let

$$
\dot{H}^{s}=\left\{F \in H^{-1}: \sum_{i=1}^{\infty} \mu_{i}^{-s}\left\langle F, \widetilde{\psi}_{i}\right\rangle^{2}<\infty\right\}
$$

with the natural norm

$$
\|F\|_{\dot{H}^{s}}=\left(\sum_{i=1}^{\infty} \mu_{i}^{-s}\left\langle F, \widetilde{\psi}_{i}\right\rangle^{2}\right)^{1 / 2}
$$

Note that the definition of these spaces immediately implies that $T: \dot{H}^{s} \rightarrow \dot{H}^{s+2}$ is an isometry for any real $s$. Proposition 4.1 below shows that $\dot{H}^{s}$ and $\widetilde{H}^{s}$ coincide for $s \in[-1,1]$ and their norms are equivalent.

To get the equivalence to extend for $s>1$, we require two additional conditions:

(c) There is an $0<\alpha \leq 1$ such that $T$ is a bounded map of $\widetilde{H}^{-1+\alpha}(\Omega)$ into $\widetilde{H}^{1+\alpha}(\Omega)$.

(d) Let $L: H^{1}(\Omega) \rightarrow H^{-1}(\Omega)$ be defined by

$$
\langle L u, \phi\rangle=A(u, \phi), \quad \text { for all } \phi \in H_{0}^{1}(\Omega) .
$$

We assume that the restriction of $L$ is a bounded operator from $H^{1+\alpha}(\Omega)$ to $\widetilde{H}^{-1+\alpha}(\Omega)$.

The first is an elliptic regularity result which can be found in, e.g., [23, 1, 10, 27]. The second is somewhat simpler related result. Both depend on the smoothness of the coefficients defining $\langle\cdot, \cdot\rangle_{1}$.

We illustrate the proof of $(\mathrm{d})$ in the case of the Dirichlet form $(a(x)=1)$. For $u \in H^{2}(\Omega)$ and $\phi \in H_{0}^{1}(\Omega)$,

$$
\left|\langle L u, \phi\rangle_{0}\right|=|A(u, \phi)|=|(-\Delta u, \phi)| \leq\|u\|_{H^{2}(\Omega)}\|\phi\|_{L^{2}(\Omega)} .
$$

This implies that $L$ is a bounded operator from $H^{2}(\Omega)$ into $L^{2}(\Omega)$. Clearly, $L$ is a bounded operator from $H^{1}(\Omega)$ into $H^{-1}(\Omega)$. Interpolating these results gives

$$
\|L u\|_{\widetilde{H}^{-1+s}(\Omega)} \leq C\|u\|_{H^{1+s}(\Omega)}, \quad \text { for all } u \in H^{1+s}(\Omega), 1 \leq s \leq 2 .
$$

This estimate is a bit more tricky for more general $A(\cdot, \cdot)$, for example, when $A(\cdot, \cdot)$ involves jumping coefficients.

The above two assumptions imply the following proposition. We note that the case of $\alpha=1$ was given in [30].

Proposition 4.1. Assume that (a)-(b) hold. Then the spaces $\widetilde{H}^{s}(\Omega)$ and $\dot{H}^{s}$ coincide for $s \in[-1,1]$ and their norms are equivalent. If, in addition, $(c)$ and $(d)$ hold, then the above equivalence extends to $s \in[1,1+\alpha]$. 
Proof. As discussed earlier, $\left\{\psi_{i}\right\}, i=1,2, \ldots$ is an orthonormal basis for $H^{-1}$ with associated eigenvalues $\left\{\mu_{i}\right\}$ and we recall the definition $\widetilde{\psi}_{i}:=\mu_{i}^{1 / 2} \psi_{i}$. This implies that $F \in H^{-1}$ if and only if the series

$$
\sum_{i=1}^{\infty}\left\langle F, \psi_{i}\right\rangle_{-1} \psi_{i}
$$

converges (to $F$ ) in $H^{-1}$, but

$$
\sum_{i=1}^{\infty}\left\langle F, \psi_{i}\right\rangle_{-1}^{2}=\sum_{i=1}^{\infty} \mu_{i}^{2}\left\langle F, \psi_{i}\right\rangle^{2}=\sum_{i=1}^{\infty} \mu_{i}\left\langle F, \widetilde{\psi}_{i}\right\rangle^{2}
$$

This shows that $H^{-1}$ and $\dot{H}^{-1}$ coincide and have identical norms.

It is clear that $\left\{\widetilde{\psi}_{i}\right\}$ is an orthonormal set in $H^{0}$. Note that if $w \in H^{0}$ satisfies $\left\langle w, \widetilde{\psi}_{i}\right\rangle_{0}=\mu_{i}^{1 / 2}\left\langle w, \psi_{i}\right\rangle=0$ for all $i$, then the identified functional is zero in $H^{-1}$ and hence

$$
\langle w, \phi\rangle_{0}=0, \quad \text { for all } \phi \in H^{1} .
$$

The density of $H^{1}$ in $H^{0}$ implies that $w=0$, i.e., $\left\{\widetilde{\psi}_{i}\right\}$ is an orthonormal basis for $H^{0}$. As above, it follows that $H^{0}$ and $\dot{H}^{0}$ coincide and have identical norms.

The argument for $H^{1}$ is essentially the same as for $H^{0}$ except the $H^{1}$-orthonormal basis is now of the form $\left\{\mu_{i} \psi_{i}\right\}, i=1,2, \ldots$. This implies that $H^{1}$ and $\dot{H}^{1}$ coincide and have identical norms. The proposition then follows for $s \in[-1,1]$ by interpolation.

We finally extend the result to $[1,1+\alpha]$ when (c) and (d) hold. Suppose that $u$ is in $\widetilde{H}^{1+\alpha}(\Omega)$. Then

$$
u=\sum_{i=1}^{\infty}\left\langle u, \psi_{i}\right\rangle_{-1} \psi_{i}
$$

where the series converges, at least, in $H^{-1}$. Now, $T L u=u$ for $u \in \widetilde{H}^{1+\alpha}(\Omega) \subset$ $H_{0}^{1}(\Omega)$ so

$$
\mu_{i}\left\langle L u, \widetilde{\psi}_{i}\right\rangle=\mu_{i}\left\langle u, \widetilde{\psi}_{i}\right\rangle_{1}=\left\langle u, T \widetilde{\psi}_{i}\right\rangle_{1}=\left\langle u, \widetilde{\psi}_{i}\right\rangle
$$

According to (d) and the first part of this proof, $L u \in \widetilde{H}^{-1+\alpha}(\Omega)=\dot{H}^{-1+\alpha}$ hence

$$
\sum_{i=1}^{\infty} \mu_{i}^{1-\alpha}\left\langle L u, \widetilde{\psi}_{i}\right\rangle^{2}=\sum_{i=1}^{\infty} \mu_{i}^{-1-\alpha}<u, \widetilde{\psi}_{i}>^{2}<\infty
$$

i.e., $u \in \dot{H}^{1+\alpha}$. Moreover,

$$
\|u\|_{\dot{H}^{1+\alpha}}=\|L u\|_{\dot{H}^{-1+\alpha}}=\|L u\|_{\widetilde{H}^{-1+\alpha}(\Omega)} \leq C\|u\|_{\widetilde{H}^{1+\alpha}(\Omega)} .
$$

Alternatively, if $u$ is in $\dot{H}^{1+\alpha}$, then $L u$ is in $\dot{H}^{-1+\alpha}=\widetilde{H}^{-1+\alpha}(\Omega)$. Now $u$ solves

$$
A(u, \phi)=<L u, \phi>, \quad \text { for all } \phi \in H^{1}
$$

and hence (c) implies that $u$ is in $\widetilde{H}^{1+\alpha}(\Omega)$ and satisfies

$$
\|u\|_{\widetilde{H}^{1+\alpha}(\Omega)} \leq C\|L u\|_{\widetilde{H}^{-1+\alpha}(\Omega)}=C\|L u\|_{\dot{H}^{-1+\alpha}}=C\|u\|_{\dot{H}^{1+\alpha}} .
$$

This shows that the spaces are identical at $s=1+\alpha$.

We note that the spaces $\widetilde{H}^{1+s}(\Omega)$ for $s \in(0, \alpha)$ are the intermediate spaces on the interpolation scale between $\widetilde{H}^{1}(\Omega)$ and $\widetilde{H}^{1+\alpha}(\Omega)$. This result is a consequence of Lemma A1 of [20] (see also Lemma A2 there). The projector needed for the application of Lemma A1[20] is $P=T L$ in our context and its stability on $H^{1+\alpha}(\Omega)$ 
is a consequence of (c) and (d). Thus, the result for $s \in(1,1+\alpha)$ follows by interpolation and completes the proof of the proposition.

The previous result coupled with assumption (c) and a duality argument entails the following approximation estimate for $T-T_{h}$.

Corollary 4.2. Assume (a), (b) and (c) hold. Then there exists a constant $C$ independent of $h$ such that for all $f \in \dot{H}^{\alpha-1}$ there holds

$$
\left\|\left(T-T_{h}\right) f\right\|_{\dot{H}^{1-\alpha}} \leq C h^{2 \alpha}\|f\|_{\dot{H}^{\alpha-1}} .
$$

Proof. Owing to (c) we deduce that

$$
\left\|\left(T-T_{h}\right) f\right\|_{\dot{H}^{1}} \preceq h^{\alpha}\|T f\|_{\dot{H}^{1+\alpha}} \preceq h^{\alpha}\|f\|_{\dot{H}^{\alpha-1}},
$$

where Proposition 4.1 is used to obtain the last estimate. The desired estimate follows from a standard duality argument (Nitsche trick).

The following equivalence relation will be instrumental in the proof of Theorem 4.3. For $0 \leq s \leq 1$, there exists a constant $c$ independent of $h$ such that for all $v_{h} \in H_{h}$

$$
\frac{1}{c}\left\|v_{h}\right\|_{\dot{H}_{h}^{s}} \leq\left\|v_{h}\right\|_{\dot{H}^{s}} \leq c\left\|v_{h}\right\|_{\dot{H}_{h}^{s}}
$$

where

$$
\left\|v_{h}\right\|_{\dot{H}_{h}^{s}}:=\left(\sum_{i=1}^{M} \mu_{i, h}^{-s}\left\langle v_{h}, \widetilde{\psi}_{i, h}\right\rangle^{2}\right)^{1 / 2}
$$

and $\mu_{i, h}, i=1, \ldots, M$ are the eigenvalues of $T_{h}$ with corresponding $L^{2}$-orthonormal eigenfunction $\widetilde{\psi}_{i, h}$ (compare (47) with the norm on $\dot{H}^{s}$ ). The above equivalence is a well-known result in the literature on multigrid methods (see, e.g., [6, Appendix A.2]).

We are now in position to derive the following result on the space discretization error.

Theorem 4.3 (Convergence of the space-discretization). Assume that (a)-(d) and (45) hold. Set $\gamma=\alpha-\beta$ when $\alpha \geq \beta$ and $\gamma=0$ when $\alpha<\beta$. For $\delta \geq \gamma$, There exists a constant $C$ uniform in $h$ and $\delta$ such that

$$
\left\|\left(T^{\beta}-T_{h}^{\beta} \pi_{h}\right) f\right\|_{L_{2}} \leq C_{\delta, h} h^{2 \alpha}\|f\|_{\dot{H}^{2 \delta}}, \quad \text { for all } f \in \dot{H}^{2 \delta} .
$$

Here

$$
C_{\delta, h}=\left\{\begin{aligned}
C \ln (1 / h): & \text { when } \delta=\gamma \text { and } \alpha \geq \beta, \\
C: & \text { when } \delta>\gamma \text { and } \alpha \geq \beta, \\
C \quad: & \text { when } \beta>\alpha .
\end{aligned}\right.
$$

Remark 4.1 (Less regularity: $\delta<\alpha-\beta$ ). If $f \in \dot{H}^{2 \delta}$ with $\delta<\alpha-\beta, T^{\beta} f$ is only in $\dot{H}^{2 \beta+2 \delta}$ and hence the best possible rate is $O\left(h^{2 \beta+2 \delta}\right)$. This can essentially be recovered from the theorem. Indeed, if (c) and (d) hold for $\alpha$ then, by interpolation, they hold for any $\alpha_{0}$ in $(0, \alpha)$. Applying the theorem with $\alpha=\delta+\beta$ gives

$$
\left\|\left(T^{\beta}-T_{h}^{\beta} \pi_{h}\right) f\right\|_{L_{2}} \leq C \ln \left(h^{-1}\right) h^{2 \beta+2 \delta}\|f\|_{\dot{H}^{2 \delta}}, \quad \text { for all } f \in \dot{H}^{2 \delta} .
$$

The identity $\|T F\|_{1}=\|F\|_{-1}$ combined with (10) and (15) implies that the largest eigenvalue of $T_{h}$ is bounded by $c_{0}^{2}$. Combining this with the above theorem and Theorem 3.5 gives the following corollary. 
NUMERICAL APPROXIMATION OF FRACTIONAL POWERS OF ELLIPTIC OPERATORS21

Corollary 4.4. Assume that (a)-(d) and (45) hold. Then for $j=R, G, E$, there exists a constant $C$ uniform in $h$ and $N$ such that for all $f \in \dot{H}^{2 \delta}$

$$
\left\|\left(T^{\beta}-\mathcal{Q}_{j, h}^{\beta} \pi_{h}\right) f\right\|_{L_{2}} \leq C_{\delta, h} h^{2 \alpha}\|f\|_{\dot{H}^{2 \delta}}+B_{j}\|f\|_{L_{2}} .
$$

Here $\delta$ and $C_{\delta, h}$ are given in the above theorem and $B_{R}, B_{G}, B_{E}$ are given in Theorem 3.5 .

We introduce the following lemma which is needed for the proof of the above theorem. Its proof is given after the proof of the theorem.

Lemma 4.5. For any $\eta \in[0,(1+\alpha) / 2)$ set $\theta=(1+\alpha) / 2-\eta$. Then

$$
\left\|t^{2 \theta}\left(t^{2} I+T\right)^{-1} f\right\|_{\dot{H}^{\alpha-1}} \leq\|f\|_{\dot{H}^{2 \eta}}, \quad \text { for all } f \in \dot{H}^{2 \eta} .
$$

In addition,

$$
\left\|t^{1+\alpha}\left(t^{2} I+T_{h}\right)^{-1} f\right\|_{L^{2}} \leq\|f\|_{\dot{H}_{h}^{1-\alpha}}, \quad \text { for all } f \in H_{h} .
$$

The above results hold for all $t \geq 0$.

Proof of Theorem 4.3. We first note that

$$
\left(I-\pi_{h}\right)\left(T^{\beta}-T_{h}^{\beta} \pi_{h}\right) f=\left(I-\pi_{h}\right) T^{\beta} f
$$

so that

$\left\|\left(I-\pi_{h}\right)\left(T^{\beta}-T_{h}^{\beta} \pi_{h}\right) f\right\|_{L^{2}} \leq C h^{2 \alpha}\left\|T^{\beta} f\right\|_{H^{2 \alpha}(\Omega)} \leq C h^{2 \alpha}\left\|T^{\beta} f\right\|_{\dot{H}^{2 \alpha}} \leq C h^{2 \alpha}\|f\|_{\dot{H}^{2 \delta}}$.

Thus, we need only bound

$$
\left\|\pi_{h}\left(T^{\beta}-T_{h}^{\beta} \pi_{h}\right) f\right\|_{L^{2}} .
$$

Applying Theorem 2.1 and (46) implies

$$
\begin{aligned}
\pi_{h}\left(T^{\beta}-T_{h}^{\beta} \pi_{h}\right) f= & C_{\beta}^{-1} \pi_{h} \int_{0}^{1} t^{2 \beta-1}\left(T_{1}(t)-T_{1, h}(t)\right) f d t \\
& +C_{\beta}^{-1} \pi_{h} \int_{0}^{1} t^{1-2 \beta}\left(T_{2}(t)-T_{2, h}(t)\right) f d t \\
& =: I_{1}+I_{2} .
\end{aligned}
$$

We first consider $I_{1}$. Setting $t_{0}=h^{\alpha / \beta}$, applying (14), (16) and the triangle inequality, we obtain

$$
\begin{aligned}
\left\|\pi_{h} \int_{0}^{t_{0}} t^{2 \beta-1}\left(T_{1}(t)-T_{1, h}(t)\right) f d t\right\|_{L^{2}} & \leq 2\left(\int_{0}^{t_{0}} t^{2 \beta-1} d t\right)\|f\|_{L_{2}} \\
& =\frac{h^{2 \alpha}}{\beta}\|f\|_{L_{2}} .
\end{aligned}
$$

Hence, it remains to derive an upper bound for

$$
\left\|\pi_{h} \int_{t_{0}}^{1} t^{2 \beta-1}\left(T_{1}(t)-T_{1, h}(t)\right) f d t\right\|_{L_{2}} .
$$

It is easy to check that $T_{1}(t)=T\left(t^{2} I+T\right)^{-1}$. Similarly, defining $\left(t^{2} I+T_{h}\right)^{-1}$ to be the inverse of $t^{2} I+T_{h}$ on $L_{2}(\Omega)$, we find that $T_{1, h}(t)=T_{h}\left(t^{2} I+T_{h}\right)^{-1}=$ $\left(t^{2} I+T_{h}\right)^{-1} T_{h}$ on $L_{2}(\Omega)$. Thus,

$$
\begin{aligned}
\pi_{h}\left(T_{1}(t)-T_{1, h}(t)\right) & =\pi_{h}\left(T\left(t^{2} I+T\right)^{-1}-\left(t^{2} I+T_{h}\right)^{-1} T_{h}\right) \\
& =t^{2}\left(t^{2} I+T_{h}\right)^{-1} \pi_{h}\left(T-T_{h}\right)\left(t^{2} I+T\right)^{-1} .
\end{aligned}
$$


We first consider the case of $\alpha \geq \beta$. Note that $\gamma<(1+\alpha) / 2$ and the theorem in this case will follow for all $\delta$ if we can prove it for $\delta \in[\gamma,(1+\alpha) / 2)$ which we shall henceforth assume.

Applying (53) shows that (52) can be bounded by

$$
\begin{gathered}
\int_{t_{0}}^{1} t^{-1+2 \delta+2 \beta-2 \alpha}\left[\left\|t^{1+\alpha}\left(t^{2} I+T_{h}\right)^{-1} \pi_{h}\right\|_{\dot{H}^{1-\alpha} \rightarrow L_{2}}\left\|\left(T-T_{h}\right)\right\|_{\dot{H}^{\alpha-1} \rightarrow \dot{H}^{1-\alpha}}\right. \\
\left.\left\|t^{1+\alpha-2 \delta}\left(t^{2} I+T\right)^{-1}\right\|_{\dot{H}^{2 \delta} \rightarrow \dot{H}^{\alpha-1}}\right] d t\|f\|_{\dot{H}^{2 \delta}} .
\end{gathered}
$$

Corollary 4.2 shows that

$$
\left\|\left(T-T_{h}\right)\right\|_{\dot{H}^{\alpha-1} \rightarrow \dot{H}^{1-\alpha}} \preceq h^{2 \alpha}
$$

while the first part of Lemma 4.5 (with $\eta=\delta \in[\gamma,(1+\alpha) / 2) \subseteq[0,(1+\alpha) / 2)$ ) implies that the third norm of (54) is uniformly bounded. Appying the second part of Lemma 4.5 and the equivalence of norms (48) gives

$$
\left\|t^{1+\alpha}\left(t^{2} I+T_{h}\right)^{-1} \pi_{h} f\right\|_{L^{2}} \leq\left\|\pi_{h} f\right\|_{\dot{H}_{h}^{1-\alpha}} \leq C\left\|\pi_{h} f\right\|_{\dot{H}^{1-\alpha}} .
$$

Since $\pi_{h}$ is bounded as an operator from $L_{2}(\Omega)$ to $L_{2}(\Omega)$ and from $H_{0}^{1}(\Omega)$ to $H_{0}^{1}(\Omega)$ due to the quasi-uniformity (45) of the triangulations, it follows by interpolation that $\pi_{h}$ is also bounded from $\dot{H}^{1-\alpha}$ to $\dot{H}^{1-\alpha}$. Combining this with (56) shows that the first norm of (54) is also uniformly bounded. It follows that when $\alpha \geq \beta$,

$$
\begin{array}{rlr}
\left\|I_{1}\right\|_{L_{2}} & \leq \frac{h^{2 \alpha}}{\beta}\|f\|_{L_{2}}+C h^{2 \alpha} \int_{t_{0}}^{1} t^{-1+2 \delta-2 \gamma} d t\|f\|_{\dot{H}^{2 \delta}} \\
& \leq\left\{\begin{array}{cr}
C h^{2 \alpha} \ln \left(h^{-1}\right)\|f\|_{\dot{H}^{2 \delta}}: & \text { when } \delta=\gamma \\
C h^{2 \alpha}\|f\|_{\dot{H}^{2 \delta}}: & \text { when } \delta>\gamma .
\end{array}\right.
\end{array}
$$

We next bound $I_{1}$ when $\alpha<\beta$. In this case, we bound (52) by

$$
\begin{aligned}
\int_{t_{0}}^{1} t^{-1+2 \beta-2 \alpha} & {\left[\left\|t^{1+\alpha}\left(t^{2} I+T_{h}\right)^{-1} \pi_{h}\right\|_{\dot{H}^{1-\alpha} \rightarrow L_{2}}\left\|\left(T-T_{h}\right)\right\|_{\dot{H}^{\alpha-1} \rightarrow \dot{H}^{1-\alpha}}\right.} \\
& \left.\left\|t^{1+\alpha}\left(t^{2} I+T\right)^{-1}\right\|_{L_{2} \rightarrow \dot{H}^{\alpha-1}}\right] d t\|f\|_{L_{2}} .
\end{aligned}
$$

We use the second part of Lemma 4.5 for the first term, (55) for the middle term and the first part of Lemma 4.5 with $\eta=0$ for the for the last. The bound

$$
\left\|I_{1}\right\|_{L^{2}} \preceq h^{2 \alpha}\|f\|_{L^{2}}
$$

follows immediately.

Finally, we derive a bound for $\left\|I_{2}\right\|_{L_{2}}$. Since $T_{2}(t):=t^{-2} T_{1}\left(t^{-1}\right)$ and $T_{2, h}(t):=$ $t^{-2} T_{1, h}\left(t^{-1}\right)$, we use (53) to compute

$$
\begin{aligned}
\pi_{H}\left(T_{2}(t)-T_{2, h}(t)\right) & =t^{-2} \pi_{h}\left(T_{1}\left(t^{-1}\right)-T_{1, h}\left(t^{-1}\right)\right) \\
& =t^{-4}\left(t^{-2} I+T_{h}\right)^{-1} \pi_{h}\left(T-T_{h}\right)\left(t^{-2} I+T\right)^{-1} \\
& =\left(I+t^{2} T_{h}\right)^{-1} \pi_{h}\left(T-T_{h}\right)\left(I+t^{2} T\right)^{-1} .
\end{aligned}
$$

We clearly have

$$
\left\|\left(I+t^{2} T_{h}\right)^{-1} \pi_{h}\right\|_{L_{2} \rightarrow L_{2}} \leq 1 \quad \text { and } \quad\left\|\left(I+t^{2} T\right)^{-1}\right\|_{L_{2} \rightarrow L_{2}} \leq 1 .
$$


Combining this with Corollary 4.2 gives

$$
\begin{gathered}
\left\|\pi_{h}\left(T_{2}(t)-T_{2, h}(t)\right)\right\|_{L_{2}} \leq\left[\left\|\left(I+t^{2} T_{h}\right)^{-1} \pi_{h}\right\|_{L_{2} \rightarrow L_{2}}\left\|T-T_{h}\right\|_{L_{2} \rightarrow L_{2}}\right. \\
\left.\left\|\left(I+t^{2} T\right)^{-1}\right\|_{L_{2} \rightarrow L_{2}}\right] \preceq h^{2 \alpha} .
\end{gathered}
$$

Hence

$$
\left\|I_{2}\right\|_{L_{2}} \preceq h^{2 \alpha} \int_{0}^{1} t^{1-2 \beta} d t\|f\|_{L_{2}} \preceq h^{2 \alpha}\|f\|_{L_{2}} .
$$

The theorem follows by gathering the estimates for $\left\|I_{1}\right\|_{L_{2}}$ and $\left\|I_{2}\right\|_{L_{2}}$.

Remark 4.2. It is only necessary to break the estimate for $I_{1}$ into integrals over $\left[0, t_{0}\right]$ and $\left[t_{0}, 1\right]$ when $\alpha \geq \beta$ and $\delta=\gamma$. In the remainder of the cases, the argument bounding $\left[t_{0}, 1\right]$ can be used to bound the integral over $[0,1]$.

Remark 4.3 (Comparison with [13]). The estimate of [13] is that

$$
\left\|\left(T^{\beta}-T_{h}^{\beta} \pi_{h}\right) f\right\|_{L_{2}} \leq C h^{2 \beta}\|f\|_{L_{2}}, \quad \text { for all } f \in L_{2},
$$

holds when $\alpha=1$. This is easily obtained from the above proof by taking $t_{0}=h$ and $\delta=0$ in which case

$$
\left\|I_{1}\right\|_{L_{2}} \leq \frac{h^{2 \beta}}{\beta}\|f\|_{L_{2}}+C h^{2 \alpha} \int_{h}^{1} t^{-1+2 \beta-2 \alpha} d t\|f\|_{L_{2}} \leq C h^{2 \beta}\|f\|_{L_{2}} .
$$

Proof of Lemma 4.5. For the first inequality of the lemma, we note that $0 \leq \eta<$ $(1+\alpha) / 2$ implies $0<\theta \leq 1$. Let $\varphi \in \dot{H}^{2 \eta}$ and let $c_{i}=\left\langle\varphi, \widetilde{\psi}_{i}\right\rangle_{0}$ so that $\varphi=$ $\sum_{i=1}^{\infty} c_{i} \widetilde{\psi}_{i}$. The definition of the norm in $\dot{H}^{\alpha-1}$ implies that

$$
\left\|t^{2 \theta}\left(t^{2}+T\right)^{-1} \varphi\right\|_{\dot{H}^{\alpha-1}}^{2}=\sum_{i=1}^{\infty}\left(\frac{t^{2 \theta} \mu_{i}^{\eta+(1-\alpha) / 2}}{t^{2}+\mu_{i}}\right)^{2} \mu_{i}^{-2 \eta} c_{i}^{2} .
$$

Note that $\theta+\eta+(1-\alpha) / 2=1$. Now $\theta=1$ only if $\alpha=1$ and $\eta=0$. In this case, the fraction in the parenthesis above is $\left(t^{2} /\left(t^{2}+\mu_{i}\right)\right)$ which is clearly bounded by one. For $\theta \in(0,1)$, a Young's inequality with $\frac{1}{p}=\theta$ and $\frac{1}{q}=\eta+(1-\alpha) / 2$ gives

$$
\frac{t^{2 \theta} \mu_{i}^{\eta+(1-\alpha) / 2}}{t^{2}+\mu_{i}} \leq \frac{1}{p} \frac{t^{2}}{t^{2}+\mu_{i}}+\frac{1}{q} \frac{\mu_{i}}{t^{2}+\mu_{i}} \leq 1
$$

Thus,

$$
\left\|t^{2 \theta}\left(t^{2} I+T\right)^{-1} \varphi\right\|_{\dot{H}^{\alpha-1}}^{2} \leq \sum_{i=1}^{\infty} \mu_{i}^{-2 \eta} c_{i}^{2}=\|\varphi\|_{\dot{H}^{2 \eta}}^{2} .
$$

This proves the first inequality of the lemma.

The second inequality is similar. Indeed, expanding $f=\sum_{i=1}^{M} c_{i, h} \widetilde{\psi}_{i, h}$ with $c_{i, h}=\left\langle f, \widetilde{\psi}_{i, h}\right\rangle_{0}$, we find

$$
A^{2}:=\left\|t^{1+\alpha}\left(t^{2} I+T_{h}\right)^{-1} f\right\|_{L_{2}}^{2}=\sum_{i=1}^{M}\left(\frac{t^{1+\alpha}}{t^{2}+\mu_{i, h}}\right)^{2} c_{i, h}^{2} .
$$

Thus,

$$
A^{2}=\sum_{i=1}^{M}\left(\frac{t^{1+\alpha} \mu_{i, h}^{\frac{1-\alpha}{2}}}{t^{2}+\mu_{i, h}}\right)^{2} \mu_{i, h}^{\alpha-1} c_{i, h}^{2}
$$


The fraction in the parenthesis is clearly bounded by one when $\alpha=1$. For $\alpha \in(0,1)$, a Young's inequality with $\frac{1}{p}=\frac{1+\alpha}{2}$ and $\frac{1}{q}=\frac{1-\alpha}{2}$ yields

$$
\left|\frac{t^{1+\alpha} \mu_{i, h}^{\frac{1-\alpha}{2}}}{t^{2}+\mu_{i, h}}\right| \leq \frac{1}{p} \frac{t^{2}}{t^{2}+\mu_{i, h}}+\frac{1}{q} \frac{\mu_{i, h}}{t^{2}+\mu_{i, h}} \leq \frac{1}{p}+\frac{1}{q}=1
$$

It follows that $A \leq\|f\|_{\dot{H}_{h}^{1-\alpha}}$ and completes the proof of the lemma.

4.1. Numerical Illustration. We consider the unit square $\Omega=(0,1)^{2} \subset \mathbb{R}^{2}$ and study numerically the efficiency in the approximation of $u$ the solution of

$$
(-\Delta)^{\beta} u=f, \quad \text { in } \Omega, \quad u=0 \quad \text { on } \partial \Omega
$$

where $f: \Omega \rightarrow \mathbb{R}$ is given for all $\left(x_{1}, x_{2}\right) \in \Omega$ by

$$
f\left(x_{1}, x_{2}\right)= \begin{cases}1 & \text { if }\left(x_{1}-0.5\right)\left(x_{2}-0.5\right)>0 \\ 0 & \text { otherwise. }\end{cases}
$$

Notice that $f \in \dot{H}^{1 / 2-\epsilon}(\Omega)$ for all $\epsilon>0$ (but not $\epsilon=0$ ) and consequently Theorem 4.3 and Remark 4.1 predict an error decay proportional to

$$
\ln (1 / h) h^{2 s}
$$

with

$$
s= \begin{cases}1, & \text { if } \beta>\frac{3}{4} \\ 2\left(\beta+\frac{1}{4}\right), & \text { otherwise. }\end{cases}
$$

The errors $\left\|u-u_{h}^{N}\right\|_{L_{2}}$ are computed using the first 300 modes in $x$ and $y$ (90000 modes) of the Fourier representation of the exact solution and the number of quadrature points is taken large enough not to influence the space discretization error. Four meshes are used to compute three observed rate of convergence

$$
\text { OROC }_{i}:=\ln \left(e_{i} / e_{i+1}\right) / \ln \left(h_{i} / h_{i+1}\right), \quad i=1,2,3,
$$

where $e_{i}$ are the $L_{2}$ errors and $h_{i}$ the diameter of the quasi-uniform subdivision $i$. The average of the three observed rate of convergence $A R O C$ are reported in Table 4.1 and are comparable with the rate predicted by Theorem 4.3 (see, Remark 4.1).

\begin{tabular}{c|c|c|c|c|c|c|c||c|c} 
& \multicolumn{7}{|c||}{$\beta<\frac{3}{4}$} & \multicolumn{2}{c}{$\beta>\frac{3}{4}$} \\
\cline { 2 - 10 } & 0.1 & 0.2 & 0.3 & 0.4 & 0.5 & 0.6 & 0.7 & 0.8 & 0.9 \\
\hline AROC & 0.92 & 1.06 & 1.22 & 1.4 & 1.52 & 1.72 & 1.86 & 1.94 & 1.96 \\
THM & 0.7 & 0.9 & 1.1 & 1.3 & 1.5 & 1.7 & 1.9 & 2.0 & 2.0
\end{tabular}

TABLE 4. Average observed rate of convergence (AROC) for different values of $\beta$ compared with the rates predicted by Theorem 4.3 (THM) ignoring the log term.

Figure 1 depicts the decay of the error $\left\|u-u_{h}^{N}\right\|_{L_{2}}$ for different values of $\beta$.

The solution for $\beta=0.1,0.5$ and 0.8 are depicted in figure 2 together with a cut over the horizontal line $\{y=0.25\}$. 


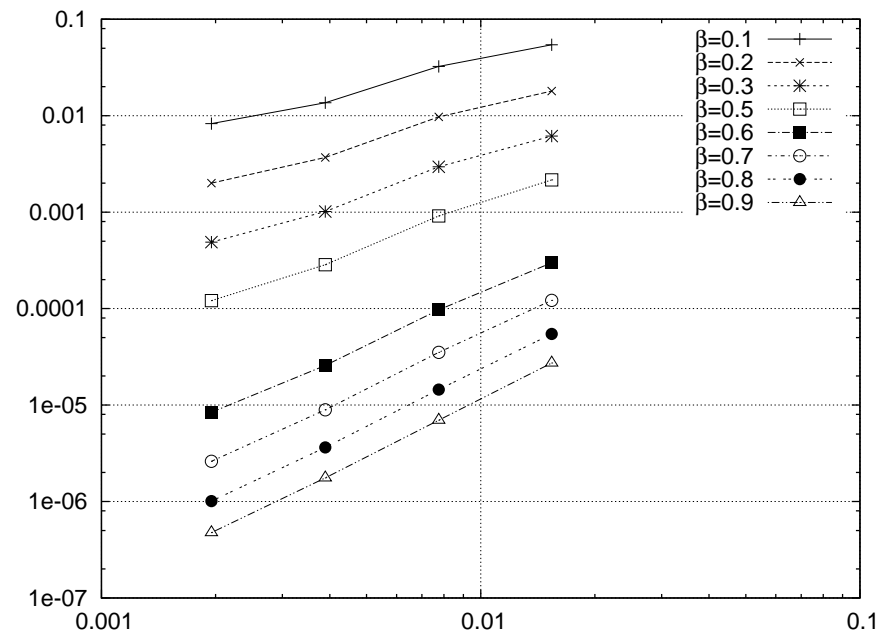

Figure $1 . L_{2}$-errors for different values of $\beta$ versus the approximate mesh size diameter used for the computations. The number of quadrature points is taken large enough not to influence the errors. The corresponding average observed rate of convergence are reported in Table 4.1 .

\section{ACKNOWLEDGMENT}

This work was supported in part by award number KUS-C1-016-04 made by King Abdulla University of Science and Technology (KAUST). The first author was also supported in part by the National Science Foundation through Grant DMS-1254618 while the second was also supported in part by the National Science Foundation through Grant DMS-1216551. In addition, the authors would like to thank E. Otarola, R.H. Nochetto and A. Salgado for fruitful discussions on the subject.

\section{REFERENCES}

[1] Constantin Bacuta, James H. Bramble, and Joseph E. Pasciak. New interpolation results and applications to finite element methods for elliptic boundary value problems. East-West J. Numer. Math., 3:179-198, 2001.

[2] Oleg G. Bakunin. Turbulence and diffusion. Springer Series in Synergetics. Springer-Verlag, Berlin, 2008. Scaling versus equations.

[3] V. Balakrishnan. Fractional powers of closed operators and the semi-groups generated by them. Pacific J. Math., 10:419-437, 1960.

[4] Peter W. Bates. On some nonlocal evolution equations arising in materials science. In Nonlinear dynamics and evolution equations, volume 48 of Fields Inst. Commun., pages 13-52. Amer. Math. Soc., Providence, RI, 2006.

[5] M. Sh. Birman and M. Z. Solomjak. Spectral theory of selfadjoint operators in Hilbert space. Mathematics and its Applications (Soviet Series). D. Reidel Publishing Co., Dordrecht, 1987. Translated from the 1980 Russian original by S. Khrushchëv and V. Peller.

[6] J.H. Bramble and X. Zhang. The analysis of multigrid methods. In Handbook of numerical analysis, Vol. VII, Handb. Numer. Anal., VII, pages 173-415. North-Holland, Amsterdam, 2000.

[7] Luis Caffarelli and Luis Silvestre. An extension problem related to the fractional Laplacian. Comm. Partial Differential Equations, 32(7-9):1245-1260, 2007. 



Figure 2. Values of $(-\Delta)^{-\beta} f$ when $f$ is the checkerboard function (57) for $\beta=0.5$ (top left), $\beta=0.8$ (top right) and $\beta=0.1$ (bottom left). The difference in shape and magnitude is highlighted in the bottom right plots which depicts the values of these function along the line $\{y=0.25\} \quad(\beta=0.1$ dashed line, $\beta=0.5$ doted line, $\beta=0.8$ solid line).

[8] P Carr, H Geman, DB Madan, and M Yor. The fine structure of asset returns: An empirical investigation. JOURNAL OF BUSINESS, 75(2):305-332, APR 2002.

[9] Peter Constantin and Jiahong Wu. Behavior of solutions of 2D quasi-geostrophic equations. SIAM J. Math. Anal., 30(5):937-948, 1999.

[10] M. Dauge. Elliptic Boundary Value Problems on Corner Domains. Lecture Notes in Mathematics, 1341, Springer-Verlag, 1988.

[11] G. Duvaut and J.-L. Lions. Inequalities in mechanics and physics. Springer-Verlag, Berlin, 1976. Translated from the French by C. W. John, Grundlehren der Mathematischen Wissenschaften, 219.

[12] A. Cemal Eringen. Nonlocal continuum field theories. Springer-Verlag, New York, 2002.

[13] Firoshi Fujita and Takashi Suzuki. Evolution Problems. Handbook of Numerical Analysis, II, Finite element methods. Part 1 (Ciarlet, P. G. and Lions, J.-L., eds.). North-Holland, Amsterdam, 1991.

[14] Ivan P. Gavrilyuk. An algorithmic representation of fractional powers of positive operators. Numer. Funct. Anal. Optim., 17(3-4):293-305, 1996.

[15] Ivan P. Gavrilyuk, Wolfgang Hackbusch, and Boris N. Khoromskij. H-matrix approximation for the operator exponential with applications. Numerische Mathematik, 92:83-111, 2002.

[16] Ivan P. Gavrilyuk, Wolfgang Hackbusch, and Boris N. Khoromskij. Data-sparse approximation to the operator-valued functions of elliptic operator. Math. Comp., 73(247):1297-1324, 2004. 
[17] Ivan P. Gavrilyuk, Wolfgang Hackbusch, and Boris N. Khoromskij. Data-sparse approximation to a class of operator-valued functions. Math. Comp., 74(250):681-708, 2005.

[18] Ivan P. Gavrilyuk, Wolfgang Hackbusch, and Boris N. Khoromskij. Hierarchical tensorproduct approximation to the inverse and related operators for high-dimensional elliptic problems. Computing, 74(2):131-157, 2005.

[19] Guy Gilboa and Stanley Osher. Nonlocal operators with applications to image processing. Multiscale Model. Simul., 7(3):1005-1028, 2008.

[20] J.-L. Guermond. The LBB condition in fractional Sobolev spaces and applications. IMA J. Numer. Anal., 29(3):790-805, 2009.

[21] M. Ilic, F. Liu, I. Turner, and V. Anh. Numerical approximation of a fractional-in-space diffusion equation. I. Fract. Calc. Appl. Anal., 8(3):323-341, 2005.

[22] M. Ilic, F. Liu, I. Turner, and V. Anh. Numerical approximation of a fractional-in-space diffusion equation. II. With nonhomogeneous boundary conditions. Fract. Calc. Appl. Anal., 9(4):333-349, 2006.

[23] R.B. Kellogg. Interpolation between subspaces of a hilbert space. Technical report, Univ. of Maryland,, Inst. Fluid Dynamics and App. Math., Tech. Note BN-719, 1971.

[24] John Lund and Kenneth L. Bowers. Sinc methods for quadrature and differential equations. Society for Industrial and Applied Mathematics (SIAM), Philadelphia, PA, 1992.

[25] B. M. McCay and M. N. L. Narasimhan. Theory of nonlocal electromagnetic fluids. Arch. Mech. (Arch. Mech. Stos.), 33(3):365-384, 1981.

[26] William McLean and Vidar Thomée. Iterative solution of shifted positive-definite linear systems arising in a numerical method for the heat equation based on Laplace transformation and quadrature. ANZIAM J., 53(2):134-155, 2011.

[27] Sergey Nazarov and Boris Plamenevsky. Elliptic problems in domains with piecewise smooth boundaries. De Gruyter expositions in mathematics, De Gruyter, 1994.

[28] R.H. Nochetto, E. Otarola, and A.J. Salgado. A PDE approach to fractional diffusion in general domains. part I: A priori error analysis. submitted.

[29] S. A. Silling. Reformulation of elasticity theory for discontinuities and long-range forces. $J$. Mech. Phys. Solids, 48(1):175-209, 2000.

[30] Vidar Thomée. Galerkin finite element methods for parabolic problems. Springer series in computational mathematics. Springer, New York, 1997.

[31] Qianqian Yang, Ian Turner, Fawang Liu, and Milos Ilić. Novel numerical methods for solving the time-space fractional diffusion equation in two dimensions. SIAM J. Sci. Comput., 33(3):1159-1180, 2011.

[32] Toshio Yoshida. Functional Analysis. Springer-Verlag, New York, 1995.

Department of Mathematics, Texas A\&M University, College Station, TX 778433368.

E-mail address: bonito@math.tamu.edu

Department of Mathematics, Texas A\&M University, College Station, TX 778433368 .

E-mail address: pasciak@math.tamu.edu 